\title{
Propagation and overturning of three-dimensional Boussinesq wave packets with rotation
}

\author{
Alain D. Gervais $\odot,{ }^{1, *}$ Quinlan Ede, ${ }^{2}$ Gordon E. Swaters $\odot,{ }^{1}$ Ton S. van den Bremer $\odot,{ }^{3}$ \\ and Bruce R. Sutherland ${ }^{2,4}$ \\ ${ }^{1}$ Department of Mathematical and Statistical Sciences, University of Alberta, Edmonton, \\ Alberta T6G 2G1, Canada \\ ${ }^{2}$ Department of Earth and Atmospheric Sciences, University of Alberta, Edmonton, Alberta T6G 2E3, Canada \\ ${ }^{3}$ Department of Engineering Science, University of Oxford, Oxford OX1 3PJ, United Kingdom \\ ${ }^{4}$ Department of Physics, University of Alberta, Edmonton, Alberta T6G 2E1, Canada
}

(Received 7 July 2020; accepted 23 March 2021; published 12 April 2021)

\begin{abstract}
The stability and overturning of fully three-dimensional internal gravity wave packets is examined for a rotating, uniformly stratified Boussinesq fluid that is stationary in the absence of waves. We derive through perturbation theory an integral expression for the mean flow induced by upward-propagating fully localized wave packets subject to Coriolis forces. This induced Bretherton flow manifests as a dipolelike recirculation about the wave packet in the horizontal plane. We perform numerical simulations of fully localized wave packets with the predicted Bretherton flow superimposed, for a range of initial amplitudes, wave-packet aspect ratios, and relative vertical wave numbers spanning the hydrostatic and nonhydrostatic regimes. Results are compared with predictions based on linear theory of wave breaking due to overturning, convection, self-acceleration, and shear instability. We find that nonhydrostatic wave packets tend to destabilize due to self-acceleration, eventually overturning although the initial amplitude is well below the overturning amplitude predicted by linear theory. Strongly hydrostatic waves, propagating almost entirely in the horizontal, are found not to attain amplitudes sufficient to become shear unstable, overturning instead due to localized steepening of isopycnals. Results are discussed in the broader context of previous studies of one- and two-dimensional wave packets overturning and recent observations of oceanic internal waves.
\end{abstract}

DOI: 10.1103/PhysRevFluids.6.044801

\section{INTRODUCTION}

Internal gravity waves propagate within stably stratified fluids, transporting energy and irreversibly depositing momentum where they break. Even without breaking, moderately large amplitude wave packets induce a mean flow that can act nonlinearly to feedback on the waves themselves, which in turn can alter the height at which waves begin to overturn [1,2]. Wave packets induce qualitatively distinct mean flows depending on whether the wave packet is (1) streamwise-periodic, vertically localized, and spanwise-infinite, (2) streamwise- and vertically localized and spanwise-infinite, or (3) localized in all three spatial dimensions. Wave packets are thereby characterized as being one-, two-, or three-dimensional, respectively. The mean flow induced by one-dimensional wave packets is streamwise-uniform, unidirectional, and concentrated in the vertical region occupied by the vertically propagating wave packet. This mean flow results from the vertical divergence of momentum flux. An explicit expression for this mean flow in

*Corresponding author: adgervai@ualberta.ca 
a Boussinesq fluid was derived using conservation of momentum for quasimonochromatic wave packets [2], being equivalent to the pseudomomentum of internal waves [3,4]. Two-dimensional wave packets without rotation similarly induce a mean flow resulting from a divergent momentum flux. Unlike in the one-dimensional case, the induced mean flow is itself divergent and so, for the fluid to remain incompressible, an order amplitude-squared pressure gradient is established. It has been shown [5-7] that the induced mean flow manifests as long, hydrostatic waves that propagate far streamwise and vertically below an upward-propagating wave packet. Conversely, two-dimensional wave packets induce an evanescent long wave response [8] if the Coriolis parameter is of greater magnitude than the forcing frequency (set by the ratio of the wave packet's vertical group speed to its vertical scale of modulation). Three-dimensional wave packets again induce qualitatively distinct mean flows [5-7]. In particular, the induced mean flow-referred to as the Bretherton flow-results from forcing by the nonlinear terms in the momentum equations acting upon the vertical component of vorticity, and manifests as a horizontal recirculation about the wave packet, being positive through the middle of the wave packet, and negative on its spanwise flanks [5,9].

Interactions between gravity waves and their induced mean flow can lead to nonlinear amplitude growth or decay, overturning, and eventually turbulent breaking. In essence, the wave-induced mean flow, acting through the weakly nonlinear dispersion relation, Doppler-shifts the wave frequency, resulting in a local change to the group velocity. Local increase (decrease) of the group velocity leads to wave spreading (accumulation), a characteristic manifestation of modulational stability (instability). As a result, the onset of overturning of one-dimensional waves is delayed (hastened), so waves overturn above (below) the height predicted by linear theory [10,11] (see also Whitham [12]). For one-dimensional waves not influenced by rotation, the transition between the modulationally stable and unstable regimes occurs at a relative vertical wave number (the ratio of the magnitudes of the vertical to horizontal wave numbers of a monochromatic wave with amplitude modulated by a height-dependent envelope) of $2^{-1 / 2} \approx 0.7$, corresponding to wave packets propagating with the fastest vertical group speed.

Two-dimensional wave packets, unlike their one-dimensional counterparts, are always modulationally unstable, as the induced mean flow (long waves) is positive above and negative below the center of the wave packet [13]. Sutherland [14] heuristically formulated a prediction for the amplitude at which Boussinesq wave packets ultimately overturn due to self-acceleration, according to the wave-induced mean flow exceeding the streamwise group velocity. The agreement was found to be good in simulations of one-dimensional but not two-dimensional wave packets.

A study of three-dimensional wave packets subject to Coriolis effects was conducted by Tabaei and Akylas [6], who focused primarily on wave packets that were either round (for which the wave packet extents were comparable in all three dimensions) and strongly nonhydrostatic, twodimensional (having infinite spanwise extent, but comparable streamwise and vertical extents), or flat (for which the horizontal wave packet extents were both much larger than the vertical extent). In the work by van den Bremer and Sutherland [9] (henceforth vdBS18), a similar study was conducted, though neglecting Coriolis effects. Their study focused, in part, on understanding the transition between qualitatively different induced mean flows as a wave packet's spanwise extent broadened, transitioning from three- to effectively two-dimensional. The purpose of this paper is to build on the work of Sutherland [14] and of vdBS18 to compare with linear theory the overturning times of fully three-dimensional finite-amplitude wave packets in a uniformly stratified, rotating Boussinesq fluid with no background flow. Our study differs in scope from both Tabaei and Akylas [6] and vdBS18 in that we derive an explicit integral formula for the Bretherton flow subject to rotation, and examine through fully nonlinear numerical simulations a broader range of nonhydrostatic and more strongly hydrostatic waves. One aspect of our investigation is to characterize the overturning time in terms of various hydrodynamic instability mechanisms affecting internal gravity wave evolution.

The paper is laid out as follows. The equations for the mean flows induced by three-dimensional wave packets are derived in Sec. II, followed by the introduction of critical amplitudes corresponding to the onset of various wave instabilities. Details of the numerical model used to simulate the 
evolution of the wave packets are presented in Sec. III. The results of simulations for a variety of wave packet parameters are shown in Sec. IV. Section V discusses the results and offers some concluding remarks.

\section{THEORY}

Here we derive an integral expression for the Bretherton flow induced by a three-dimensional wave packet in a uniformly stratified Boussinesq fluid, subject to the Coriolis force but ignoring diffusion and viscous effects. We adapt the procedure of vdBS18, who derived the corresponding expression for the Bretherton flow without rotation, and in so doing we also identify and reconcile an error by the latter in which a small number of terms were omitted during their derivation. We then derive, using linear theory, predictions of critical wave amplitudes for the onset of static instability (overturning), instability for which convection can develop within a wave period, self-acceleration, and shear.

\section{A. Wave-induced mean flow}

The governing equations are the Boussinesq Euler equations, the internal energy equation (expressed in terms of vertical displacement), and the equation of mass conservation for an incompressible fluid, given, respectively, by

$$
\begin{aligned}
\frac{D \mathbf{u}}{D t}-f_{0} v \hat{\mathbf{e}}_{x}+f_{0} u \hat{\mathbf{e}}_{y} & =-\frac{1}{\rho_{0}} \nabla p-N_{0}^{2} \xi \hat{\mathbf{e}}_{z}, \\
\frac{D \xi}{D t} & =w, \\
\nabla \cdot \mathbf{u} & =0 .
\end{aligned}
$$

The equations are given in Cartesian coordinates $\mathbf{x}=(x, y, z)$ with corresponding unit vectors $\hat{\mathbf{e}}_{x}, \hat{\mathbf{e}}_{y}, \hat{\mathbf{e}}_{z}$, in which $z$ is vertical. Here $D / D t=\partial_{t}+\mathbf{u} \cdot \nabla$ is the material derivative, in which $\mathbf{u}=$ $(u, v, w)$ is the velocity vector, $f_{0}$ is the Coriolis parameter, $p$ is the dynamic pressure, $g$ is the acceleration of gravity, $\rho_{0}$ is a constant reference density, $\bar{\rho}(z)$ is the background density, and $\xi$ is the vertical displacement. The fluid is assumed to be uniformly stratified so the squared buoyancy frequency $N_{0}^{2}=-\left(g / \rho_{0}\right) d \bar{\rho} / d z$ is constant. The fluctuation density can thus be expressed in terms of $\xi$ by $\rho=-\xi d \bar{\rho} / d z$.

An explicit formula for the Bretherton flow, $\mathbf{u}_{\mathrm{BF}}$, is found assuming quasimonochromatic wave packets. Without loss of generality, the waves are assumed to propagate in the $x z$ plane, with wave number vector $\mathbf{k}=(k, 0, m)$. For convenience, we assume wave packets propagate forward in $x$ and upward in $z$, so $k>0$ and $m<0$. The corresponding frequency, given by the dispersion relation, is $\omega=\left(N_{0}^{2} k^{2}+f_{0}^{2} m^{2}\right)^{1 / 2} /\|\mathbf{k}\|$, in which $\|\mathbf{k}\|=\left(k^{2}+m^{2}\right)^{1 / 2}$.

We define a slow timescale $T$ and slow spatial variables, $\tilde{\mathbf{X}} \equiv(\tilde{X}, \tilde{Y}, \tilde{Z})$, in a frame translating at the group velocity of the wave packet, $\mathbf{c}_{g}=\left(c_{g x}, 0, c_{g z}\right)=k m\left[N_{0}^{2}-f_{0}^{2}\right](m, 0,-k) /\left(\omega\|\mathbf{k}\|^{4}\right)$. Explicitly, $\tilde{X}=\epsilon_{x}\left(x-c_{g x} t\right), \tilde{Y}=\epsilon_{y} y, \tilde{Z}=\epsilon_{z}\left(z-c_{g z} t\right)$, and $T=\epsilon^{2} t$, where $\epsilon_{x}=1 /\left(k \sigma_{x}\right), \epsilon_{y}=$ $1 /\left(k \sigma_{y}\right)$, and $\epsilon_{z}=1 /\left(k \sigma_{z}\right)$ are inversely related to the wave packet extent, $\sigma_{x}, \sigma_{y}$, and $\sigma_{z}$, in their respective directions. For quasimonochromatic wave packets, it is assumed that $\epsilon=\max \left\{\epsilon_{x}, \epsilon_{y}, \epsilon_{z}\right\} \ll$ 1. The slow variable $T$ describes the timescale for dispersion of the wave packet, which acts at order $\epsilon^{2}$. Consequently, dispersive effects are effectively ignored. For notational convenience, we define $(X, Y, Z) \equiv\left(x-c_{g x} t, y, z-c_{g z} t\right)$, and it is understood that $X, Y$, and $Z$ derivatives are of order $\epsilon_{x}$, $\epsilon_{y}$, and $\epsilon_{z}$, respectively.

With this notation, the leading-order representation of a field, $\eta$, associated with a quasimonochromatic wave packet is given by

$$
\eta_{0}^{(1)}(\mathbf{x}, t) \equiv \frac{1}{2} A_{\eta}(\mathbf{X}, T) e^{i(\mathbf{k} \cdot \mathbf{x}-\omega t)}+\text { c.c. },
$$

where the subscript and superscript on $\eta$ denote, respectively, the field's order in $\epsilon$ and in amplitude, and c.c. denotes the complex conjugate. At leading order, the various wave fields are related by 
TABLE I. Expressions for the amplitude envelopes of various fields as they relate to the amplitude envelope, $A$, of the vertical displacement field. The center column contains the leading-order (wave-scale) relationships and the right column contains their respective first-order (envelope-scale) corrections (with respect to $\epsilon$ ). Subscripts on $A$ denote partial derivatives, $\beta_{x}$ is a constant given by Eq. (9a), and we have defined $B \equiv 1+k \beta_{x}$ and $K \equiv 1+k^{2} / m^{2}$ for convenience. By convention, the actual fields are taken to be the real parts of $e^{i(\mathbf{k} \cdot \mathbf{x}-\omega t)}$ times the tabulated expressions.

\begin{tabular}{lcc}
\hline \hline Field & $O\left(\alpha^{1} \epsilon^{0}\right)$ & $O\left(\alpha^{1} \epsilon^{1}\right)$ \\
\hline Vertical displacement & $\xi_{0}^{(1)}=A$ & $\xi_{1}^{(1)}=i \beta_{x}\left\{A_{X}-\frac{k}{m} A_{Z}\right\}$ \\
Streamwise velocity & $u_{0}^{(1)}=i \omega \frac{m}{k} A$ & $u_{1}^{(1)}=\frac{m}{k}\left\{\left(c_{g x}-\frac{\omega}{k}-\omega \beta_{x}\right) A_{X}+i \frac{f_{0}}{k} A_{Y}\right.$ \\
& & $\left.+\left(c_{g z}+\frac{\omega}{m}-\omega \frac{k}{m} \beta_{x}\right) A_{Z}\right\}$ \\
Spanwise velocity & $v_{0}^{(1)}=f_{0} \frac{m}{k} A$ & $v_{1}^{(1)}=i \frac{m}{k^{2}}\left\{f_{0} B A_{X}-i \omega A_{Y}-f_{0} \frac{k}{m} B A_{Z}\right\}$ \\
Vertical velocity & $w_{0}^{(1)}=-i \omega A$ & $w_{1}^{(1)}=-\left\{\left(c_{g x}-\omega \beta_{x}\right) A_{X}+\left(c_{g z}+\frac{k}{m} \omega \beta_{x}\right) A_{Z}\right\}$ \\
Streamwise vorticity & $\zeta_{x 0}^{(1)}=-i f_{0} \frac{m^{2}}{k} A$ & $\zeta_{x 1}^{(1)}=\frac{m^{2}}{k^{2}}\left\{f_{0} B A_{X}-i \omega K A_{Y}-f_{0} \frac{k}{m}(1+B) A_{Z}\right\}$ \\
Spanwise vorticity & $\zeta_{y 0}^{(1)}=-\omega \frac{\|\mathbf{k}\|^{2}}{k} A$ & $\zeta_{y 1}^{(1)}=i \frac{m^{2}}{k}\left\{\left[K\left(c_{g x}-\omega \beta_{x}\right)-\frac{\omega}{k}\right] A_{X}+i \frac{f_{0}}{k} A_{Y}\right.$ \\
& & $\left.+\left[K\left(c_{g z}+\frac{k}{m} \omega \beta_{x}\right)+\frac{\omega}{m}\left(2+\frac{k}{m}\right)\right] A_{Z}\right\}$ \\
Vertical vorticity & $\zeta_{z 0}^{(1)}=i f_{0} m A$ & $\zeta_{z 1}^{(1)}=-f_{0}\left\{m \beta_{x} A_{X}-B A_{Z}\right\}$ \\
\hline \hline
\end{tabular}

the polarization relations for plane waves, given in the center column of Table I. In the table, the nondimensional amplitude is represented by $\alpha \equiv k A_{0}$, in which $A_{0}=\|A\|$ is the maximum vertical displacement of the waves in the wave packet and we have defined $A \equiv A_{\xi}$ for convenience. Alternatively, we could represent nondimensional amplitude as $\alpha_{0}=\left|m A_{0}\right|$, which would be a more direct measure of the amplitude relative to that of overturning waves (for which $\left|m A_{0}\right|=1$ ). For the derivation presented here, the particular choice of definition is unimportant. To obtain polarization relations at the next order in $\epsilon$ (accounting for the finite wave packet extent), it is necessary to impose the structure of one field. Without loss of generality, we choose to impose the structure of the vertical displacement field at both leading and first orders, as this is a physically intuitive quantity. Hence we write $\xi=\xi_{0}^{(1)}+\xi_{1}^{(1)}$, in which $\xi_{0}^{(1)}=\frac{1}{2} A e^{i \varphi}+$ c.c. and $\xi_{1}^{(1)}=i \frac{1}{2}\left(\beta_{x} A_{X}+\beta_{y} A_{Y}+\beta_{z} A_{Z}\right) e^{i \varphi}+$ c.c., where $\varphi=k x+m z-\omega t$. The values of $\beta_{x}, \beta_{y}$, and $\beta_{z}$ are to be determined. The resulting $O\left(\alpha^{1} \epsilon^{1}\right)$ polarization relations are given in the right column of Table I.

For three-dimensional wave packets that are not too wide, the Eulerian induced mean flow, $\mathbf{u}_{\mathrm{BF}}$, is primarily horizontal, being governed by the evolution equation for vertical vorticity and requiring the horizontal motion is divergence free [5,9]. From the vertical component of the curl of Eq. (1a), we have

$$
\partial_{t t}\left(\partial_{x} v_{\mathrm{BF}}-\partial_{y} u_{\mathrm{BF}}\right)=\underbrace{\overline{\partial_{t}\left\{\zeta \cdot \nabla w-\nabla \cdot\left(\zeta_{z} \mathbf{u}\right)\right\}}}_{\equiv F_{z}},
$$

subject to $\partial_{x} u_{\mathrm{BF}}+\partial_{y} v_{\mathrm{BF}}=0$. The right-hand side represents forcing by self-interaction of the waves within the packet on the scale of the wave packet. The overline denotes averaging over the fast wave scales, leaving only the slowly varying mean flow terms. The response to the forcing gives rise to an order amplitude-squared induced mean flow, $\mathbf{u}_{\mathrm{BF}}$. Neglected on the left-hand side of Eq. (3) is the term $-f_{0} \partial_{t z} w_{\mathrm{BF}}$, which is effectively ignored as $\left|w_{\mathrm{BF}}\right| \ll\left|u_{\mathrm{BF}}\right|,\left|v_{\mathrm{BF}}\right|$ for three-dimensional wave packets $[5,6,9,15-18]$.

Following vdBS18, the Bretherton flow is decomposed into the sum of physically distinct contributions,

$$
\mathbf{u}_{\mathrm{BF}}=\mathbf{u}_{\mathrm{DF}}+\mathbf{u}_{\mathrm{RF}}
$$


Here, $\mathbf{u}_{\mathrm{DF}}$ is the divergent-flux induced flow and $\mathbf{u}_{\mathrm{RF}}$ is the response flow. The divergent-flux induced flow arises from the slow variations in the nonlinear advection terms in the momentum Eq. (1a), and is given implicitly by

$$
\frac{\partial \mathbf{u}_{\mathrm{DF}}}{\partial t}=\overline{-\nabla \cdot\left(\mathbf{u}^{(1)} \otimes \mathbf{u}^{(1)}\right)}
$$

where $\otimes$ is the tensor product. An expression for the time evolution of $\mathbf{u}_{\mathrm{DF}}$ is found by substituting the leading-order polarization relations into the right-hand side of Eq. (5), and computing the mean of each term. This operation amounts to ignoring the superharmonic terms, proportional to $e^{ \pm 2 i \varphi}$, in each product on the right-hand side of Eq. (5), leaving only the slowly varying mean contributions. As a result, we find that

$$
\frac{\partial \mathbf{u}_{\mathrm{DF}}}{\partial t}=-\frac{1}{2} \frac{m^{2}}{k^{2}}\left(\omega^{2}\left(\partial_{X}-\frac{k}{m} \partial_{Z}\right)|A|^{2}, f_{0}^{2} \partial_{Y}|A|^{2},-\omega^{2} \frac{k}{m}\left(\partial_{X}-\frac{k}{m} \partial_{Z}\right)|A|^{2}\right),
$$

where $|\cdot|$ denotes the modulus. Unlike vdBS18, we do not immediately proceed to solve this for the components of $\mathbf{u}_{\mathrm{DF}}$, as it is convenient to defer this until we solve for the components of $\mathbf{u}_{\mathrm{BF}}$ itself.

An explicit expression for the forcing given by $F_{z}$ in Eq. (3) is found by substituting the polarization relations into $F_{z}$ and extracting the terms for which the product of the complex exponentials cancels. As a result, we find that the leading-order nonzero forcing arises at $O\left(\alpha^{2} \epsilon^{3}\right)$ :

$$
\begin{aligned}
\left(F_{z}\right)_{3}^{(2)}= & \frac{1}{2} \frac{\partial}{\partial t}\left\{\omega^{2} \frac{m^{2}}{k^{2}}\left[\partial_{X Y}-\frac{k}{m} \partial_{Y Z}\right]|A|^{2}-f_{0}^{2} \frac{m^{2}}{k^{2}} \partial_{X Y}|A|^{2}\right\} \\
& -\frac{1}{2} \frac{\partial}{\partial t}\left\{\left[\frac{m^{2}}{k^{2}}\left(\omega^{2}+f_{0}^{2}\right)+\omega^{2} \frac{\|\mathbf{k}\|^{2}}{k} \beta_{x}\right] A \partial_{X Y} A+\omega^{2} \frac{\|\mathbf{k}\|^{2}}{k} \beta_{y} A \partial_{Y Y} A\right. \\
& \left.-\left[\frac{m}{k}\left(\omega^{2}+f_{0}^{2}\right)-\omega^{2} \frac{\|\mathbf{k}\|^{2}}{k} \beta_{z}\right] A \partial_{Y Z} A\right\} .
\end{aligned}
$$

Using Eq. (6), it can be shown that the first expression on the right-hand side of Eq. (7) is equal to $\partial_{t t}\left(\nabla \times \mathbf{u}_{\mathrm{DF}}\right) \cdot \hat{\mathbf{e}}_{z}$. The remaining expressions in Eq. (7) were erroneously omitted by vdBS18 (who also had $f_{0}=0$ ), although it will be shown that certain values of $\beta_{x}, \beta_{y}$, and $\beta_{z}$ lead this omission to be of no consequence to their results.

We then substitute Eq. (7) into the right-hand side of Eq. (3) and use Eq. (4) on the right-hand side of Eq. (3), canceling the $\partial_{t t}\left(\nabla \times \mathbf{u}_{\mathrm{DF}}\right)$ term on both sides to yield

$$
\begin{aligned}
\partial_{t t}\left(\nabla \times \mathbf{u}_{\mathrm{RF}}\right) \cdot \hat{\mathbf{e}}_{z}= & -\frac{1}{2} \frac{\partial}{\partial t}\left\{\left[\frac{m^{2}}{k^{2}}\left(\omega^{2}+f_{0}^{2}\right)+\omega^{2} \frac{\|\mathbf{k}\|^{2}}{k} \beta_{x}\right] A \partial_{X Y} A\right. \\
& \left.+\omega^{2} \frac{\|\mathbf{k}\|^{2}}{k} \beta_{y} A \partial_{Y Y} A-\left[\frac{m}{k}\left(\omega^{2}+f_{0}^{2}\right)-\omega^{2} \frac{\|\mathbf{k}\|^{2}}{k} \beta_{z}\right] A \partial_{Y Z} A\right\} .
\end{aligned}
$$

The horizontally irrotational nature [5] of the response flow $\mathbf{u}_{\mathrm{RF}}$ imposes the values of $\beta_{x}, \beta_{y}$, and $\beta_{z}$ so the right-hand side of Eq. (8) is zero. Explicitly,

$$
\begin{aligned}
& \beta_{x}=-\frac{m^{2}}{k\|\mathbf{k}\|^{2}}\left(1+\frac{f_{0}^{2}}{\omega^{2}}\right), \\
& \beta_{y}=0, \\
& \beta_{z}=-\frac{k}{m} \beta_{x} .
\end{aligned}
$$

In the absence of rotation, $\beta_{x}$ and $\beta_{z}$ reduce to $-c_{g x} / \omega$ and $-c_{g z} / \omega$, respectively. Hence, the horizontal irrotationality of $\mathbf{u}_{\mathrm{RF}}$ implies that the previously mentioned omitted terms in vdBS18 
vanish identically so there is no change to their results. With these considerations, Eq. (3) becomes

$$
-\mathbf{c}_{g} \cdot \nabla\left(\partial_{X} v_{\mathrm{BF}}-\partial_{Y} u_{\mathrm{BF}}\right)=\partial_{X}\left(\partial_{t} v_{\mathrm{DF}}\right)-\partial_{Y}\left(\partial_{t} u_{\mathrm{DF}}\right),
$$

in which we have used $\partial_{t} \approx-\mathbf{c}_{g} \cdot \nabla$ on the left-hand side, and $\partial_{t} u_{\mathrm{DF}}$ and $\partial_{t} v_{\mathrm{DF}}$ are given in terms of spatial derivatives of $|A|^{2}$ in Eq. (6).

Equation (10) is coupled with the incompressibility condition for the Bretherton flow, $\partial_{X} u_{\mathrm{BF}}+$ $\partial_{Y} v_{\mathrm{BF}}=0$. The resulting system is solved using 3D Fourier transforms with respect to $\mathbf{X}=$ $(X, Y, Z)$ to convert the system of partial differential equations to one of algebraic equations. The Fourier transform is defined here by $\hat{\eta}(\boldsymbol{\kappa}) \equiv(2 \pi)^{-3} \int_{\mathbb{R}^{3}} \eta(\mathbf{X}) e^{-i(\kappa \cdot \mathbf{X})} d X d Y d Z$, where $\boldsymbol{\kappa}=(\kappa, \lambda, \mu)$ is the vector of transform variables. Solving the resulting system of equations and inverse Fourier transforming, we obtain

$$
\left[\begin{array}{c}
u_{\mathrm{BF}} \\
v_{\mathrm{BF}}
\end{array}\right]=\frac{1}{2} \frac{m^{2}}{k^{2}} \int_{\mathbb{R}^{3}} \frac{\lambda}{\left\|\boldsymbol{\kappa}^{\perp}\right\|^{2}}\left(\frac{\omega^{2}}{c_{g x}}-f_{0}^{2} \frac{\kappa}{\mathbf{c}_{g} \cdot \boldsymbol{\kappa}}\right)\left[\begin{array}{c}
\lambda \\
-\kappa
\end{array}\right] \widehat{|A|^{2}} e^{i(\kappa \cdot \mathbf{X})} d \kappa d \lambda d \mu,
$$

in which $\left\|\boldsymbol{\kappa}^{\perp}\right\|^{2}=\kappa^{2}+\lambda^{2}$. This represents the solution to a special case of a Poisson equation for the streamfunction of the Bretherton flow, Eq. (9.29) in Bühler and McIntyre [15] in the limit of no background flow or beta effects, the latter derived by applying generalized Lagrangian-mean theory in a potential vorticity framework. Equation (11) likewise represents the solution to Eq. (22) in Tabaei and Akylas [6]. In the absence of rotation, this solution reduces to that found by vdBS18, given by their Eq. (3.15). Rotation gives rise to a key dynamical difference between the induced mean flow represented by Eq. (11) compared to its nonrotating analog, namely, that the rotating Bretherton flow is not restricted to the isopycnals intersected by the wave packet. This is implied by dependence of the solution on $\mu$ that is intractable from $\kappa$ and $\lambda$ dependence of $\widehat{|A|^{2}}$ on the right-hand side of Eq. (11), unlike in the case with $f_{0}=0$. The influence of rotation is also made more explicit in Eq. (9.29) of Ref. [15], in which the response to the nonlinear forcing includes explicitly the vertical gradient of a vertical displacement term when $f_{0} \neq 0$.

In the simulations that follow, solutions of Eq. (11) are considered for a Gaussian wave packet with amplitude envelope given by

$$
A=A_{0} \exp \left[-\frac{1}{2}\left(\frac{X^{2}}{\sigma_{x}^{2}}+\frac{Y^{2}}{\sigma_{y}^{2}}+\frac{Z^{2}}{\sigma_{z}^{2}}\right)\right] .
$$

The Fourier transform of the square of Eq. (12) is

$$
\widehat{|A|^{2}}=\frac{1}{8 \pi^{3 / 2}} \sigma_{x} \sigma_{y} \sigma_{z} A_{0}^{2} \exp \left[-\left(\sigma_{x}^{2} \kappa^{2}+\sigma_{y}^{2} \lambda^{2}+\sigma_{z}^{2} \mu^{2}\right) / 4\right] .
$$

This result is substituted into Eq. (11), which is then solved numerically.

Slices at $z=0$ of the streamwise component of the Bretherton flow are shown in color in Fig. 1 for round, long, and wide wave packets. In each case, the flow was induced by a wave packet initialized with a peak vertical displacement amplitude of $A_{0}=0.08 k^{-1}$ and a relative vertical wave number of $m / k=-11.4$, corresponding to hydrostatic waves propagating at an angle of $\Theta=\tan ^{-1}(|m / k|)=85^{\circ}$ to the vertical. The Coriolis parameter was taken to be $0.05 N_{0}$ so $\omega / f_{0}=2.01$. Superimposed in black in each panel are 20 streamlines, $\tilde{\psi}_{\mathrm{BF}}=\psi_{\mathrm{BF}} /\left(N_{0} / k^{2}\right)$, defined implicitly by $u_{\mathrm{BF}} \equiv-\partial_{y} \psi_{\mathrm{BF}}$ and $v_{\mathrm{BF}} \equiv \partial_{x} \psi_{\mathrm{BF}}$ and separated by fixed contour intervals. As found with no rotation [5,9], the dipolelike horizontal structure of the Bretherton flow manifests clearly in the streamlines about each wave packet. The weakest initial peak value of the Bretherton flow is associated with wide wave packets: As the spanwise aspect ratio $R_{y}=\sigma_{y} / \sigma_{x}$ increases, the magnitude of the Bretherton flow decreases, as anticipated from the results of vdBS18, who showed without rotation that the peak decreases as $1 / R_{y}$ (see also Bühler [1]). The effect of rotation in Fig. 1 is to broaden the area over which the magnitude of the Bretherton flow is non-negligible, although this change is not clearly detected graphically from plots with $f_{0}=0$ (not shown) analogous to those in Fig. 1. Rotation-enhanced breaking of streamline symmetry is only subtly evident for 

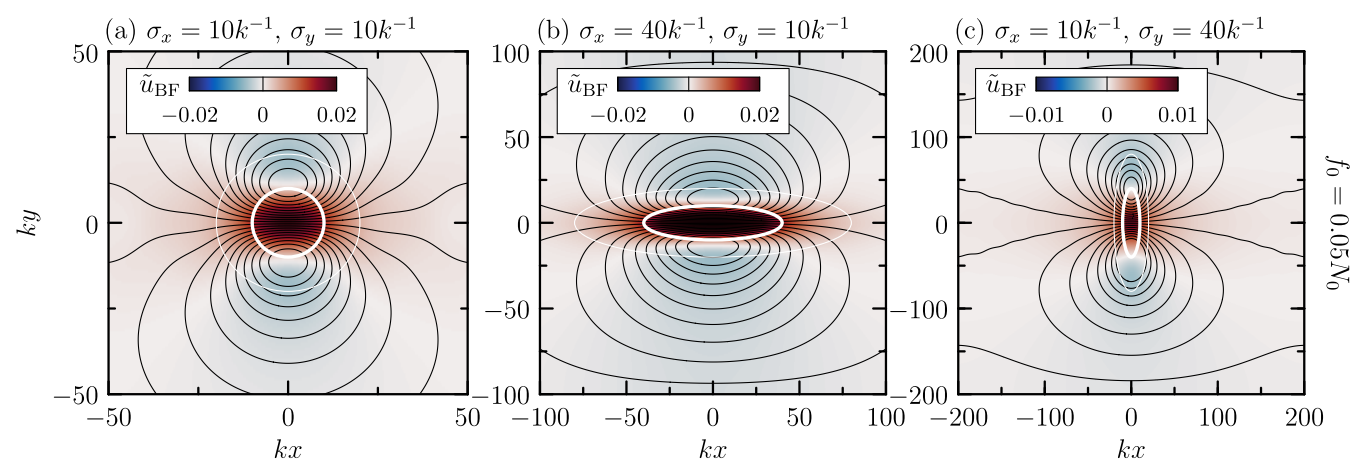

FIG. 1. Slices of the initial nondimensionalized Bretherton flow, $\tilde{u}_{\mathrm{BF}}=u_{\mathrm{BF}}\left(x, y, z_{0}, 0\right) /\left(N_{0} / k\right)$ through $z_{0}=0$, given by Eq. (11), for round (a), long (b), and wide (c) Gaussian wave packets with rotation set by $f_{0}=0.05 N_{0}$. In each case, $\sigma_{z}=10 k^{-1}$. The wave packets shown were initialized with a peak vertical displacement amplitude of $A_{0}=0.08 k^{-1}$ and a relative vertical wave number of $m / k=-11.4$. The black contours are isolines of nondimensionalized streamfunction, $\tilde{\psi}_{\mathrm{BF}}=\psi_{\mathrm{BF}}\left(x, y, z_{0}, 0\right) /\left(N_{0} / k^{2}\right)$. The heavy and light white ellipses show the first and second standard deviations, respectively, of the Gaussian wave packet.

wide wave packets [Fig. 1(c)], indicated by the elevated concentration of closed contours near $k y=50$ compared to $k y=-50$. Increasing $f_{0}$ acts also to increase the magnitude of the positive and negative regions of the Bretherton flow. Notably, as $f_{0}$ increases from $0.01 N_{0}$ to $0.05 N_{0}$, the largest contribution of rotation is an increase to $u_{\mathrm{BF}}(\mathbf{0})$ of $16 \%$ (round wave packets) and $19 \%$ (long and wide wave packets).

Comparing the magnitudes of the induced mean flows with and without rotation we find generally that the nonrotating contribution, obtained by including only the first term in the parentheses in the integrand in Eq. (11), represents the dominant contribution to the Bretherton flow. It follows that for a fixed Coriolis parameter and amplitude, $\left|u_{\mathrm{BF}}(\mathbf{0}) / A_{0}^{2}\right| \stackrel{\propto}{\sim}(m / k)^{2} \omega^{2} / c_{g x}$ increases with $|m / k|$. As a result, the peak Bretherton flow (relative to $A_{0}^{2}$ ) induced by a hydrostatic wave packet is greater than that induced by a nonhydrostatic wave packet of the same amplitude. Hence we anticipate that interactions between waves and their induced Bretherton flow play a role in the overturning of moderately large amplitude nonhydrostatic wave packets and relatively small-amplitude hydrostatic wave packets alike.

\section{B. Wave instability and critical wave amplitudes}

Following Sutherland [14], we consider several mechanisms for the breaking of internal waves, neglecting parametric subharmonic instability, as this is a relatively slow process. All critical amplitudes are normalized using the streamwise wavelength, $\lambda_{x}=2 \pi / k$, so $\tilde{A} \equiv A / \lambda_{x}$ is a nondimensional measure of vertical displacement amplitude [except for those in Fig. 2(b)].

\section{Static, convection, and self-acceleration instability}

An internal gravity wave is overturning if waves are of sufficient amplitude that dense fluid is advected over less dense fluid, hence the vertical gradient of the total density field is positive somewhere in the flow field: $\bar{\rho}^{\prime}(z)+\partial \rho(\mathbf{x}, t) / \partial z>0$. Equivalently, this is the condition that the squared total buoyancy frequency, $N_{T}^{2}$, is negative somewhere, in which $N_{T}^{2} \equiv N_{0}^{2}+\Delta N^{2}$, and $\Delta N^{2}=-N_{0}^{2} \partial \xi / \partial z$ is the local change in the squared buoyancy frequency due to waves. The static instability criterion thus becomes $\min \left\{\Delta N^{2}\right\}<-N_{0}^{2}$. The corresponding critical amplitude at which a plane wave is predicted to overturn is given nondimensionally by

$$
\tilde{A}_{\mathrm{OT}}=\frac{1}{2 \pi}\left|\frac{k}{m}\right|=\frac{1}{2 \pi} \cot \Theta,
$$



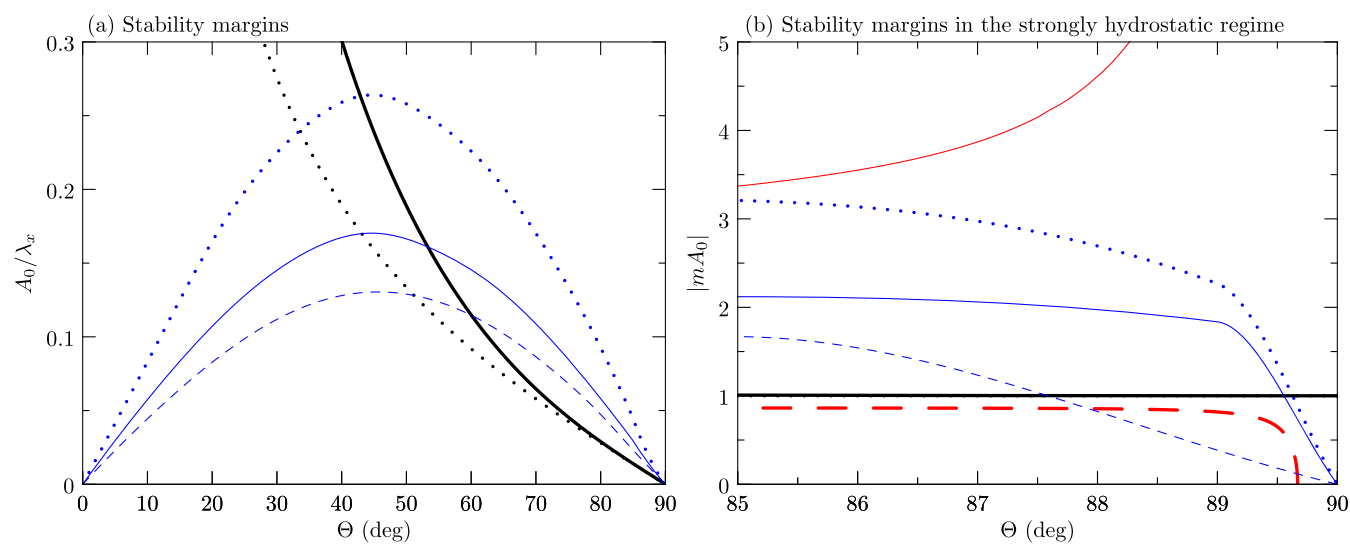

FIG. 2. Curves of marginal stability emphasizing waves in the (a) nonhydrostatic and (b) hydrostatic wavenumber ranges. The respective $\Theta$ axes correspond to waves spanning the frequency ranges $N_{0} \geqslant \omega \geqslant f_{0}$ and $8.77 \gtrsim \omega / f_{0} \geqslant 1$. The dotted and solid black curves in (a) and (b) indicate the amplitudes above which a plane internal gravity wave is predicted to be, respectively, statically and convectively unstable, given by Eqs. (14) and (15), respectively. The blue curves in (a) and (b) indicate the amplitudes above which a wave packet is predicted to be unstable owing to self-acceleration, and are given by Eq. (18) for $R_{y}=\sigma_{y} / \sigma_{x}=1$ (solid), $R_{y}=1 / 4$ (dashed) and $R_{y}=4$ (dotted). The solid and dashed red curves in (b) indicate the amplitudes above which a wave is predicted to be shear unstable using respectively our definition of the gradient Richardson number Eq. (19) and that of Fritts and Rastogi [19] and Achatz [20].

where $\Theta=\tan ^{-1}(|m / k|)$ is the angle between lines of constant phase and the vertical. Rotation has no direct influence on $\tilde{A}_{\mathrm{OT}}$, as Eq. (14) is derived using only the leading-order vertical displacement field, which depends only implicitly on rotation through dependence of the wave's phase on $\omega\left(\mathbf{k} ; f_{0}\right)$. The condition given by Eq. (14) is equivalently stated in terms of steepness of a one-dimensional plane wave, $s \equiv u / c_{p}=1$, where $c_{p} \equiv \omega / k$ is the phase speed of a monochromatic internal wave in one dimension. The curve of marginal stability corresponding to Eq. (14) is shown in Fig. 2 as the black dotted curve.

Although a statically unstable wave is prone to develop convective instabilities, whether such instabilities materialize depends on the timescale for the growth of convective instability relative to the period of the waves.

The condition that convective instability has sufficient time to overwhelm the restorative oscillatory motion of the waves is given by $\sigma_{\max }>\omega$, where $\sigma_{\max }=N_{0} \sqrt{|m| A-1}$ is the maximum instability growth rate [14]. Rearranging these two expressions and using the definition of $\omega$ yields the critical amplitude at which a plane wave is predicted to become convectively unstable, given nondimensionally by

$$
\tilde{A}_{\mathrm{CV}}=\frac{1}{2 \pi} \cot \Theta\left(1+\cos ^{2} \Theta+\frac{f_{0}^{2}}{N_{0}^{2}} \sin ^{2} \Theta\right) .
$$

This reduces to the expression given by Sutherland [14] in the case $f_{0}=0$. The curve of marginal stability corresponding to Eq. (15) is shown for $f_{0}=0.01 N_{0}$ in Figs. 2(a) and 2(b) as the solid black lines. Note that $\tilde{A}_{\mathrm{CV}}>\tilde{A}_{\mathrm{OT}}$, even as $\omega \rightarrow f_{0}\left(|\Theta| \rightarrow 90^{\circ}\right)$.

Somewhat heuristically, Sutherland [14] proposed that a wave packet is prone to instability due to self-acceleration if the waves have sufficient amplitude that their maximum induced mean flow is at least as large as the wave packet's streamwise group speed. This heuristic condition was found to be in good agreement with the results of simulations of one-dimensional but not two-dimensional wave packets [14]. For a one-dimensional wave packet without rotation, the induced mean flow is given simply by $U_{\mathrm{DF}}=N_{0} k|A|^{2} / 2$. From this, an explicit formula for the critical amplitude of 
self-acceleration is $\tilde{A}_{\mathrm{SA}}=\sin (2 \Theta) /(\pi \sqrt{8})$ [14]. In the case of a three-dimensional wave packet whose induced mean flow is governed by the Bretherton flow with maximum at $\mathbf{X}=\mathbf{0}$, it is necessary to compare $c_{g x}$ to $u_{\mathrm{BF}}(\mathbf{0})$, so generally

$$
\tilde{A}_{\mathrm{SA}}=\frac{A_{0} k}{2 \pi} \sqrt{\frac{c_{g x}}{u_{\mathrm{BF}}(\mathbf{0})}} .
$$

For a Gaussian wave packet, using Eqs. (11) and (13), we find

$$
\begin{aligned}
u_{\mathrm{BF}}(\mathbf{0})= & \frac{1}{16 \pi^{3 / 2}} \frac{m^{2}}{k^{2}} \frac{\omega^{2}}{c_{g x}} \sigma_{x} \sigma_{y} \sigma_{z} A_{0}^{2} \int_{\mathbb{R}^{3}} \frac{\lambda^{2}}{\left\|\boldsymbol{\kappa}^{\perp}\right\|^{2}} e^{-\left(\sigma_{x}^{2} \kappa^{2}+\sigma_{y}^{2} \lambda^{2}+\sigma_{z}^{2} \mu^{2}\right) / 4} d \boldsymbol{\kappa} \\
& -f_{0}^{2} \frac{1}{16 \pi^{3 / 2}} \frac{m^{2}}{k^{2}} \sigma_{x} \sigma_{y} \sigma_{z} A_{0}^{2} \int_{\mathbb{R}^{3}} \frac{\lambda^{2}}{\left\|\boldsymbol{\kappa}^{\perp}\right\|^{2}} \frac{\kappa}{\mathbf{c}_{g} \cdot \boldsymbol{\kappa}} e^{-\left(\sigma_{x}^{2} \kappa^{2}+\sigma_{y}^{2} \lambda^{2}+\sigma_{z}^{2} \mu^{2}\right) / 4} d \boldsymbol{\kappa} .
\end{aligned}
$$

The first line of Eq. (17) can be evaluated explicitly [1,9], and is equal to $\max \left\{u_{\mathrm{DF}}\right\} /\left(R_{y}+1\right)$, where $\max \left\{u_{\mathrm{DF}}\right\}=m^{2} \omega^{2} A_{0}^{2} /\left(2 k^{2} c_{g x}\right)$. The integral in the second line of Eq. (17) must be evaluated numerically. Recasting in terms of nondimensional variables, $\tilde{\kappa}=\sigma_{x} \kappa, \tilde{\lambda}=\sigma_{y} \lambda$, and $\tilde{\mu}=\sigma_{z} \mu$, it is written as $\int_{\mathbb{R}^{3}} I d \tilde{\boldsymbol{\kappa}} / c_{g x}$, where

$$
I\left(\tilde{\kappa} ; R_{y}, R_{z}, k / m\right)=\frac{\tilde{\lambda}^{2} e^{-\left(\tilde{\kappa}^{2}+\tilde{\lambda}^{2}+\tilde{\mu}^{2}\right) / 4}}{\left(R_{y}^{2} \tilde{\kappa}^{2}+\tilde{\lambda}^{2}\right)\left[1-R_{z}^{-1}(\tilde{\mu} / \tilde{\kappa})(k / m)\right]} .
$$

Here $R_{z}^{-1}=\sigma_{x} / \sigma_{z}$ is the inverse wave-packet aspect ratio in the vertical. Together with Eq. (17), the self-acceleration condition becomes

$$
\tilde{A}_{\mathrm{SA}}=\frac{k^{2}}{\sqrt{2} \pi|m|} c_{g x}\left[\frac{\omega^{2}}{R_{y}+1}-\frac{f_{0}^{2}}{8 \pi^{3 / 2}} \int_{\mathbb{R}^{3}} I\left(\tilde{\boldsymbol{\kappa}} ; R_{y}, R_{z}, k / m\right) d \tilde{\boldsymbol{\kappa}}\right]^{-1 / 2} .
$$

Profiles of $\tilde{A}_{\mathrm{SA}}$ are shown in Fig. 2(a) as the solid, dashed, and dotted blue curves corresponding to wave packets with $\sigma_{z}=10 k^{-1}$ and with spanwise aspect ratios $R_{y}=1,1 / 4$, and 4, respectively.

\section{Shear instability}

The motion of strongly hydrostatic internal gravity waves is almost entirely horizontal. As such, the waves may be subject to shear instability. The shear stability of a parallel flow is characterized by the gradient Richardson number, $\mathrm{Ri}_{g}$, defined as the ratio of the total squared buoyancy frequency to the square of the vertical shear of the streamwise velocity of the waves, $\partial u / \partial z$. If the flow is influenced by background rotation, it is necessary to incorporate into the definition of $\mathrm{Ri}_{g}$ the shear associated with $u$ and $v$. Fritts and Rastogi [19] (see also Achatz [20]) defined $\mathrm{Ri}_{g}$ using the squared magnitude of the vertical shear vector, $\left\|\left(\partial_{z} u, \partial_{z} v\right)\right\|^{2}=(\partial u / \partial z)^{2}+(\partial v / \partial z)^{2}$. Their results suggested hydrostatic waves favour the development of shear instability as opposed to convective instability.

We model the local velocity shear by considering the vertical gradient of the magnitude of the horizontal velocity vector $\left\|\mathbf{u}^{\perp}\right\|=\sqrt{u^{2}+v^{2}}$ rather than the magnitude of the shear vector. Using the leading-order polarization relations in the center column of Table I, the gradient Richardson number is then given by

$$
\mathrm{Ri}_{g} \equiv \frac{N_{0}^{2}+\Delta N^{2}}{\left(\partial\left\|\mathbf{u}^{\perp}\right\| / \partial z\right)^{2}}=\left[\frac{N_{0} k}{m\left(\omega^{2}-f_{0}^{2}\right)}\right]^{2} \frac{1}{\alpha_{0}^{2}} \frac{\left(1-\alpha_{0} \sin \varphi\right)\left[\left(\omega^{2}-f_{0}^{2}\right) \sin ^{2} \varphi+f_{0}^{2}\right]}{\sin ^{2} \varphi \cos ^{2} \varphi},
$$

in which $\alpha_{0} \equiv|m A|$ and $\varphi$ denotes the phase of the waves. The minimum value of $\mathrm{Ri}_{g}$ is found by determining the critical values of the phase, $\varphi_{c}$, for which $d \mathrm{Ri}_{g} / d \varphi=0$. This gives an implicit relation between $\varphi_{c}$ and $\alpha_{0}$ for a given $k / m$. Setting $\mathrm{Ri}_{g}=1 / 4$ in Eq. (19) as a threshold for instability [21,22] gives a second relation between $\varphi_{c}$ and $\alpha_{0}$, which can be substituted into $d \mathrm{Ri}_{g} / d \varphi=0$, 
hence giving a closed (fifth-order) polynomial for $\sin \varphi_{c}$. The roots are found numerically and from these the critical amplitude $\tilde{A}_{\mathrm{SHR}}$ is found.

The results are plotted as the solid red curve in Fig. 2(b). For comparison, this minimization procedure was also performed analytically using the definition of $\mathrm{Ri}_{g}$ of Refs. [19,20], plotted as the dashed red curve in Fig. 2(b). Because the solid red curve lies above the amplitude predicted for overturning and convection, it is predicted that hydrostatic internal gravity waves are always overturning before attaining sufficiently large vertical displacement amplitudes for shear instability to occur. This counterintuitive prediction follows alternatively from evaluating Eq. (19) for a wave on the cusp of overturning, for which $\alpha_{0}=1$ according to Eq. (14): Defining $\tilde{f}^{2} \equiv f_{0}^{2} /\left(\omega^{2}-f_{0}^{2}\right)>$ 0 and $\tilde{k}^{2} \equiv k^{2} / m^{2}$ for convenience, Eq. (19) is

$$
\mathrm{Ri}_{g}=\frac{N_{0}^{2}\left(1+\tilde{k}^{2}\right)}{N_{0}^{2}-f_{0}^{2}} \frac{1}{\alpha_{0}^{2}} \frac{\left(1-\alpha_{0} \sin \varphi\right)\left(\sin ^{2} \varphi+\tilde{f}^{2}\right)}{\sin ^{2} \varphi \cos ^{2} \varphi},
$$

having used $\omega^{2}-f_{0}^{2}=\tilde{k}^{2}\left(N_{0}^{2}-f_{0}^{2}\right) /\left(1+\tilde{k}^{2}\right)$ in the leading coefficient. It follows that

$$
\left.\mathrm{Ri}_{g}\right|_{\alpha_{0}=1}=\frac{N_{0}^{2}}{N_{0}^{2}-f_{0}^{2}} \frac{\left(1+\tilde{k}^{2}\right)\left(\sin ^{2} \varphi+\tilde{f}^{2}\right)}{\sin ^{2} \varphi(1+\sin \varphi)}>\frac{1+\tilde{k}^{2}}{1+\sin \varphi} \geqslant \frac{1}{2}\left(1+\tilde{k}^{2}\right) \geqslant \frac{1}{2},
$$

in which we used $\sin ^{2} \varphi / \sin ^{2} \varphi=1$, which remains finite even as the denominator $\sin ^{2} \varphi \rightarrow 0$, corresponding to the fact that shear tends locally to zero at the point in the phase where the wave approaches overturning. The analytical bound in Eq. (21) has been determined previously using an alternative definition of $\mathrm{Ri}_{g}$ [23]. These independent analyses suggest a strongly hydrostatic wave will remain shear stable until attaining its critical amplitude for overturning. Further amplitude growth to $\alpha_{0}>1$ causes $\mathrm{Ri}_{g}$ to fall discontinuously below zero. This apparent disparity between our predictions and those of Fritts and Rastogi [19] suggests that the shear of a strongly hydrostatic internal gravity wave influenced by rotation cannot alone induce a shear instability of sufficient strength to cause the wave to overturn [23,24]. This prediction is investigated using the results of fully nonlinear numerical simulations, described below.

\section{NUMERICS}

The three-dimensional fully nonlinear code solved the incompressible Boussinesq Navier-Stokes equations. The prognostic equations for horizontal momentum and internal energy (in which vertical displacement is used as a proxy for perturbation density) are given dimensionally by

$$
\begin{gathered}
\frac{\partial u}{\partial t}=-\frac{1}{\rho_{0}} \frac{\partial p}{\partial x}+f_{0} v-\nabla \cdot(u \mathbf{u})+v \mathscr{D} u \\
\frac{\partial v}{\partial t}=-\frac{1}{\rho_{0}} \frac{\partial p}{\partial y}-f_{0} u-\nabla \cdot(v \mathbf{u})+v \mathscr{D} v \\
\frac{\partial \xi}{\partial t}=w-\nabla \cdot(\xi \mathbf{u})+\kappa \mathscr{D} \xi
\end{gathered}
$$

The velocity vector $\mathbf{u}$, vertical displacement $\xi$, Coriolis parameter $f_{0}$, and fluctuation pressure $p$ are as in Eqs. (1a)-(1c). The system given by Eqs. (22)-(24) is closed by the inclusion of the diagnostic equations

$$
\begin{aligned}
& \frac{1}{\rho_{0}} \nabla^{2} p=-\left[\frac{\partial^{2}\left(u^{2}\right)}{\partial x^{2}}+\frac{\partial^{2}\left(v^{2}\right)}{\partial y^{2}}+\frac{\partial^{2}\left(w^{2}\right)}{\partial z^{2}}\right]-2\left[\frac{\partial^{2}(u v)}{\partial x \partial y}+\frac{\partial^{2}(u w)}{\partial x \partial z}+\frac{\partial^{2}(v w)}{\partial y \partial z}\right] \\
&+f_{0}\left(\frac{\partial v}{\partial x}-\frac{\partial u}{\partial y}\right)-N_{0}^{2} \frac{\partial \xi}{\partial z} \\
& \frac{\partial w}{\partial z}=-\frac{\partial u}{\partial x}-\frac{\partial v}{\partial y}
\end{aligned}
$$


the latter of these being equivalent to the incompressibility condition $\nabla \cdot \mathbf{u}=0$. The parameters $\nu$ and $\kappa$ are, respectively, the kinematic viscosity and thermal diffusivity. The operator $\mathscr{D}$ acts in Fourier space as a Laplacian-like diffusion operator that applies to all spanwise and vertical wave numbers but only to streamwise wave numbers greater than a viscous damping wave number, $k_{d}$. These diffusive terms were included on the right-hand sides of Eqs. (22)-(24) to assist with numerical stability by damping numerical noise while not acting to attenuate the waves. For all simulations, the diffusive terms were recast using the Reynolds and Prandtl numbers, respectively, $\operatorname{Re} \equiv N_{0} /\left(k^{2} v\right)=1000$ and $\operatorname{Pr} \equiv v / \kappa=1$, with $k_{d}=257$.

All simulations were initialized with a trivariate Gaussian wave packet whose initial vertical displacement field was prescribed at leading and first orders to be

$$
\begin{aligned}
\xi(x, y, z, 0)= & A_{0}\left\{\cos (k x+m z)+\beta_{x}\left(\frac{x}{\sigma_{x}^{2}}-\frac{k}{m} \frac{z}{\sigma_{z}^{2}}\right) \sin (k x+m z)\right\} \\
& \times \exp \left[-\frac{1}{2}\left(\frac{x^{2}}{\sigma_{x}^{2}}+\frac{y^{2}}{\sigma_{y}^{2}}+\frac{z^{2}}{\sigma_{z}^{2}}\right)\right],
\end{aligned}
$$

where $A_{0}$ was the initial maximum vertical displacement, $\sigma_{x}, \sigma_{y}$, and $\sigma_{z}$, respectively, were the streamwise, spanwise, and vertical extents of the wave packet, and the coefficient $\beta_{x}$ is defined in Eq. (9a). The vertical displacement was chosen as the initialization field because it is a physically intuitive quantity, and its Gaussian structure was chosen for convenience. The horizontal velocity field $\mathbf{u}_{0}^{\perp}=\left(u_{0}, v_{0}\right)$ was initialized using the linear theory polarization relations for $u_{0}^{(1)}$ and $v_{0}^{(1)}$ given in the center column of Table I. Superimposed onto these fields were their respective $O\left(\alpha^{1} \epsilon^{1}\right)$ corrections, given in the right column of Table I.

The initial Bretherton flow was superimposed onto the horizontal flow field using Eq. (11). It is necessary to perform this step for wave packets whose initial amplitude is sufficiently large that the waves are initially subject to the weakly nonlinear effect of interaction with the wave-induced mean flow. Neglecting to superimpose the initial predicted induced mean flow results in an "error flow" [9], an unphysical "deficit" [25], or residual flow that remains at the initial location of the wave packet, and is of equal magnitude but opposite sign of the predicted induced mean flow (see also Refs. [7,8]). Although small-amplitude wave packets initially induce a mean flow of negligible magnitude, for consistency across simulations such wave packets were nonetheless initialized to include their Bretherton flow.

The scales for time and domain size were set by fixing $N_{0}=1$ and $k=1$ (with units of inverse time and inverse length, respectively). The domain is spectral in all three spatial directions and is triply periodic. The real-space domain, $L_{x} \times L_{y} \times L_{z}$, was set to be large enough that neither the waves nor their induced mean flow self-interacted across the periodic boundaries for the duration of the simulation $\left(100 / N_{0}\right)$. The streamwise and vertical dimensions were set by specifying the number of wavelengths $n_{\mathrm{w}, x}$ and $n_{\mathrm{w}, z}$ that span $L_{x}$ and $L_{z}$, respectively, so $L_{x}=2 \pi n_{\mathrm{w}, x} / k$ and $L_{z}=2 \pi n_{\mathrm{w}, z} /|m|$. Typically, $n_{\mathrm{w}, x}=64$ and $n_{\mathrm{w}, z}=8$ for nonhydrostatic waves to $n_{\mathrm{w}, z}=128$ for the most strongly hydrostatic waves. These dimensions were discretized such that either 16 or 32 grid points spanned one wavelength. Our coordinate system was oriented such that there was no wave propagation in the spanwise direction, hence $L_{y}$ was specified directly, typically $L_{y}=200 / k$. At least $n_{y}=256$ grid points spanned $L_{y}$ for each simulation. The Fourier-space domain was discretized by $n_{x} / 2$ Fourier modes in the $x$ direction and by $n_{y}$ and $n_{z}$ Fourier modes in the $y$ and $z$ directions, respectively. Here $n_{x}$ and $n_{z}$ are set by the product of the number of wavelengths times the number of grid points per wavelength in the respective directions.

A limited series of test simulations of nonhydrostatic wave packets were run using a domain of size $2 L_{x} \times 2 L_{y} \times 2 L_{z}$ but with no change in resolution, and using a domain of size $L_{x} \times L_{y} \times L_{z}$ but with double the $x, y$, and $z$ resolution described above. Domain size was found to have no significant qualitative or quantitative effects on the results; doubling the resolution led to wave overturning at most one buoyancy period earlier than in the standard resolution simulation. The resolution of the hydrostatic simulations reported on below represents the practical maximum resolution available. 
TABLE II. Initial conditions, domain size and resolution, and output diagnostics from the subset of the fully nonlinear numerical simulations discused or explicitly mentioned in text. Columns are simulation ID, vertical displacement amplitude nondimensionalized by $k$, and as a fraction of the predicted amplitudes for static and self-acceleration instability [given by Eqs. (14) and (18), respectively, where $\tilde{A}_{0}=A_{0} / \lambda_{x}$ ], absolute relative vertical wave number $|m / k|$ and corresponding $\Theta=\tan ^{-1}|m / k|\left(\mathrm{deg}\right.$ ), spanwise aspect ratio $R_{y}=\sigma_{y} / \sigma_{x}$, domain size $L_{x} k \times L_{y} k \times L_{z} k$ and resolution $n_{x} \times n_{y} \times n_{z}$, approximate simulation runtime in core-hours, and diagnosed overturning time $N_{0} t_{\text {От }}$. IDs with a superscript - or + sign, respectively, denote simulations performed with $f_{0}=-0.05 N_{0}$ and $+0.05 N_{0}$. Simulations were run in parallel using $16,32,44$, or 48 cores.

\begin{tabular}{lcccccccccc}
\hline \hline ID & $A_{0} k$ & $\frac{\tilde{A}_{0}}{\tilde{A}_{\mathrm{OT}}}$ & $\frac{\tilde{A}_{0}}{\tilde{\mathrm{SA}}_{\mathrm{A}}}$ & $|m / k|$ & $\Theta$ & $R_{y}$ & $L_{x} k \times L_{y} k \times L_{z} k$ & $n_{x} \times n_{y} \times n_{z}$ & Runtime & $N_{0} t_{\mathrm{OT}}$ \\
\hline $\mathrm{NH} 1$ & 0.5 & 0.10 & 1.24 & 0.2 & $11^{\circ}$ & 1 & $402.1 \times 200.0 \times 251.3$ & $1024 \times 256 \times 256$ & 196 & 49 \\
$\mathrm{NH} 2$ & 0.35 & 0.07 & 0.86 & 0.2 & $11^{\circ}$ & 1 & $402.1 \times 200.0 \times 251.3$ & $1024 \times 256 \times 256$ & 332 & 111 \\
$\mathrm{NH} 3$ & 0.2 & 0.04 & 0.49 & 0.2 & $11^{\circ}$ & 1 & $402.1 \times 200.0 \times 251.3$ & $1024 \times 256 \times 256$ & 631 & - \\
$\mathrm{NH} 4$ & 0.4 & 0.28 & 0.52 & 0.7 & $35^{\circ}$ & $1 / 4$ & $402.1 \times 400.0 \times 143.6$ & $1024 \times 512 \times 512$ & 778 & 32 \\
$\mathrm{NH} 5$ & 0.5 & 0.35 & 0.65 & 0.7 & $35^{\circ}$ & $1 / 4$ & $402.1 \times 400.0 \times 143.6$ & $1024 \times 512 \times 512$ & 586 & 25 \\
$\mathrm{NH6}$ & 0.4 & 0.28 & 0.26 & 0.7 & $35^{\circ}$ & 4 & $402.1 \times 400.0 \times 143.6$ & $1024 \times 512 \times 512$ & 734 & 29 \\
$\mathrm{NH} 7$ & 0.4 & 0.28 & 0.40 & 0.7 & $35^{\circ}$ & 1 & $402.1 \times 200.0 \times 143.6$ & $1024 \times 256 \times 512$ & 264 & 32 \\
$\mathrm{NH} 8$ & 0.6 & 0.42 & 0.60 & 0.7 & $35^{\circ}$ & 1 & $402.1 \times 200.0 \times 143.6$ & $1024 \times 256 \times 512$ & 224 & 21 \\
$\mathrm{NH} 9^{-}$ & 0.2 & 0.40 & 0.24 & 2.0 & $63^{\circ}$ & 1 & $402.1 \times 200.0 \times 100.5$ & $1024 \times 256 \times 1024$ & 909 & 39 \\
$\mathrm{H} 1$ & 0.08 & 0.91 & 0.43 & 11.4 & $85^{\circ}$ & 1 & $402.1 \times 200.0 \times 70.5$ & $1024 \times 256 \times 4096$ & 4896 & 45 \\
$\mathrm{H} 1^{+}$ & 0.08 & 0.91 & 0.43 & 11.4 & $85^{\circ}$ & 1 & $402.1 \times 200.0 \times 70.5$ & $1024 \times 256 \times 4096$ & 4108 & 40 \\
$\mathrm{H} 2$ & 0.15 & 0.81 & 0.39 & 5.4 & $80^{\circ}$ & 1 & $402.1 \times 200.0 \times 74.5$ & $1024 \times 256 \times 2048$ & 1562 & 32 \\
$\mathrm{H} 3$ & 0.15 & 0.81 & 0.48 & 5.4 & $80^{\circ}$ & $1 / 4$ & $402.1 \times 400.0 \times 74.5$ & $1024 \times 512 \times 2048$ & 3200 & 32 \\
$\mathrm{H} 4$ & 0.15 & 0.81 & 0.25 & 5.4 & $80^{\circ}$ & 4 & $402.1 \times 400.0 \times 74.5$ & $1024 \times 512 \times 2048$ & 3144 & 32 \\
\hline \hline
\end{tabular}

No significant qualitative or quantitative effects were observed upon halving the time step in the standard resolution simulation.

At a given time step, the code computed the $p$ and $w$ fields using data from one step prior according the diagnostic Eqs. (25) and (26), the results of which were used to resolve the $\partial u / \partial t$, $\partial v / \partial t$, and $\partial \xi / \partial t$ fields using the prognostic Eqs. (22)-(24). The horizontal momentum and vertical displacement fields were advanced in time using a third-order accurate, low-storage WilliamsonRunge-Kutta scheme $[26,27]$. The time step was taken to be $\Delta t=0.05 N_{0}^{-1}$ for all simulations.

\section{RESULTS}

Our intent is to examine for which initial vertical displacement amplitudes localized wave packets eventually overturn, and to characterize the physical mechanisms responsible. A series of numerical simulations was performed using a range of relative vertical wave numbers, initial amplitudes, and wave-packet aspect ratios. These simulations also allowed us to diagnose the overturning time. Simulations evolved wave packets with initial amplitudes between $A_{0}=0.03 k^{-1} \approx 0.005 \lambda_{x}$ and $A_{0}=1.2 k^{-1} \approx 0.19 \lambda_{x}$ using relative vertical wave numbers between $m=-0.2 k$ and $m=-11.4 k$ (with the spanwise wave number $\ell=0$ fixed), thus spanning a range of strongly nonhydrostatic and hydrostatic upward-propagating waves. The respective relative vertical wave numbers corresponded to waves whose lines of constant phase made angles $\Theta=\tan ^{-1}(|m / k|)=11^{\circ}$ and $\Theta=85^{\circ}$ to the vertical. The Coriolis parameter for all simulations was fixed at $f_{0}=0.01 N_{0}$ (unless otherwise noted) as a typical value at midlatitudes. For all simulations, the vertical extent of the wave packet was fixed at $\sigma_{z}=10 \mathrm{k}^{-1}$. Most of the simulations reported on below are of round wave packets, for which $\sigma_{x}=\sigma_{y}=\sigma_{z}$, as this is the primary focus of the present paper. For comparison, select wave-number-amplitude combinations were simulated for long wave packets, for which $\sigma_{x}=4 \sigma_{y}=4 \sigma_{z}$, and wide wave packets, for which $\sigma_{x}=\frac{1}{4} \sigma_{y}=\sigma_{z}$. The Bretherton flow induced by round, long, and wide wave packets is shown, for example, in Fig. 1. A subset of the full suite of 

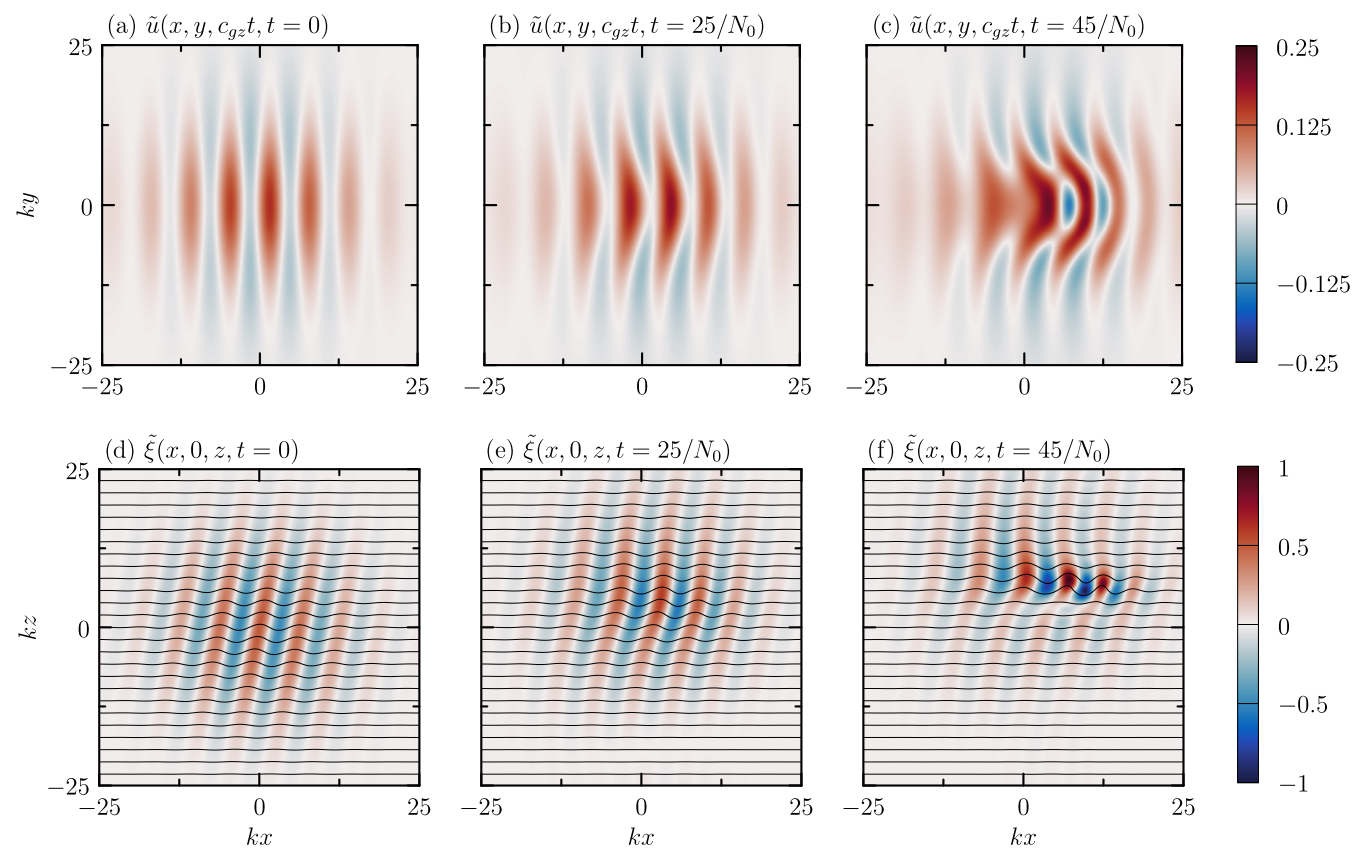

FIG. 3. Cross sections of the streamwise velocity field, $\tilde{u}=u /\left(N_{0} / k\right)$, through the horizontal plane $z=$ $c_{g z} t$ (top row) and of the vertical displacement field, $\tilde{\xi}=k \xi$, through the vertical plane $y=0$ (bottom row) of a round, moderately large amplitude nonhydrostatic internal gravity wave packet (NH1), initialized with $A_{0}=0.5 k^{-1}$ and $m=-0.2 k$. Images have been cropped to focus on the region of the domain containing the wave packet. The black curves in the bottom row represent isopycnal surfaces. The isopycnals overturned at $t=49 / N_{0}$.

simulations is discussed below; for each one, the initial conditions, domain size and resolution, and select output diagnostics are included in Table II.

\section{A. Evolution of a nonhydrostatic wave packet}

Snapshots of the evolution of a round moderately large amplitude nonhydrostatic wave packet (NH1 in Table II) with $A_{0}=0.5 k^{-1}$ and $m=-0.2 k$ are shown in Fig. 3. The top row shows cross sections of the streamwise velocity field $\tilde{u}=u /\left(N_{0} / k\right)$ through the horizontal plane $z=c_{g z} t$ and the bottom row shows cross sections of the vertical displacement field $\tilde{\xi}=k \xi$ through the vertical plane $y=0$. The times shown in each panel are representative of the wave packet at early, midlife, and near-overturning stages of its evolution. The wave packet was initially of such large amplitude as to be unstable to self-acceleration, predicted using Eq. (18). This instability is evident in Figs. 3(b) and 3(c) as crests near the center of the wave packet begin to merge with - and eventually overtake - troughs, owing to the magnitude of the induced mean flow there: $\max \left\{\left.u_{\mathrm{BF}}\right|_{t=25 / N_{0}}\right\} \approx 0.09 N_{0} / k>c_{g x} \approx 0.04 N_{0} / k$. The dipolelike structure of the Bretherton flow in the horizontal causes the symmetric deformation of the wave crests about the $k x$ axis [e.g., Fig. 3(b)]. The assumed Gaussian vertical structure of the Bretherton flow is similarly responsible for the asymmetric phase shift seen in the corresponding vertical cross section of $\tilde{\xi}$ [Fig. 3(e)]: As an individual crest propagates downward toward the vertical center of the wave packet, hence toward the region of strongest positive induced mean flow, the part of the phase nearest $\max \left\{u_{\mathrm{BF}}\right\}$ is differentially advected, resulting in an apparent steepening of phase lines just above the center of the wave packet, and a flattening of the phase lines below. Sutherland [14] observed qualitatively similar behavior in his study of one- and two-dimensional wave packets, and identified this behavior as resulting from self-acceleration. Shortly before overturning [Fig. 3(f)], the wave 

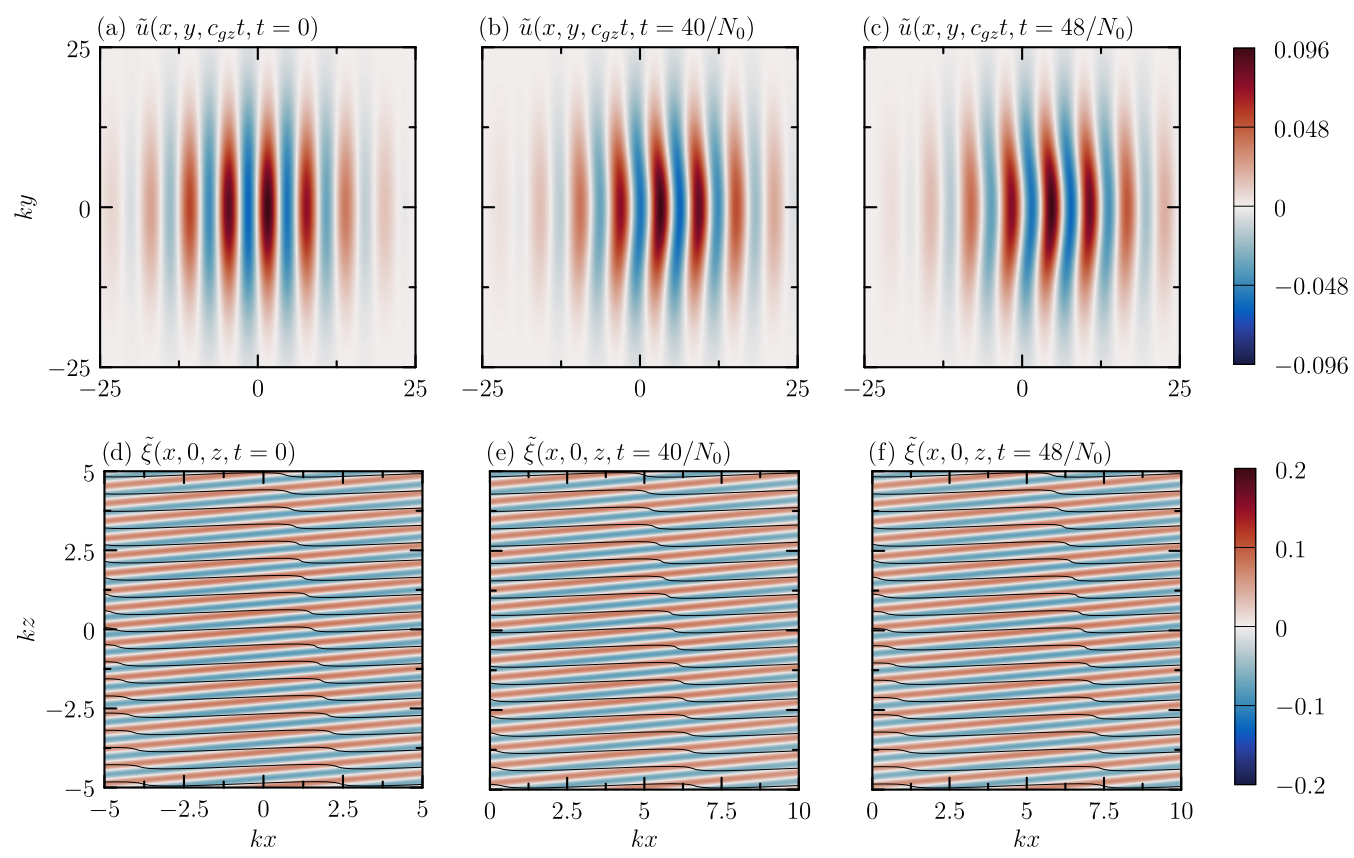

FIG. 4. As in Fig. 3 but for a round strongly hydrostatic internal gravity wave packet (H1), initialized with $A_{0}=0.08 k^{-1}$ and $m=-11.4 k$.

evolution is dominated by nonlinear effects: The peak vertical displacement has nearly doubled to $\max \left\{\left.\tilde{\xi}\right|_{t=45 / N_{0}}\right\}=0.99$ from its initial value of 0.5 , while streamwise and vertical asymmetry of isopycnal surfaces (represented by black curves) develops owing to interactions between the waves and the induced mean flow. Isopycnals near the center of the wave packet show the steepening of wave fronts as a result of nonlinear amplitude growth. The wave packet continued to evolve nonlinearly until overturning at $t=49 / N_{0}$ and ultimately breaking at $t=56 / N_{0}$.

The phase steepening and flattening behavior characterized above as resulting from selfacceleration was likewise observed for nonhydrostatic wave packets (not shown) with initial amplitudes well below that predicted for the onset of self-acceleration. The phase changes result from a local Doppler shift in wave frequency, increasing (decreasing) above (below) the center of the wave packet. Hence nonhydrostatic waves generally evolved under the effects of modulational instability (stability), and so we find that the critical amplitude for self-acceleration poorly predicted the initial amplitude for which most nonhydrostatic waves eventually overturned. Sutherland [14] similarly found that horizontal modulations of two-dimensional wave packets resulted generally in poorer agreement between the predicted amplitude for self-acceleration and simulation results (compared to the relatively good agreement found for one-dimensional wave packets).

\section{B. Evolution of strongly hydrostatic wave packets}

Snapshots of the evolution of a round strongly hydrostatic wave packet $(\mathrm{H} 1)$ with $m=-11.4 k$ and $A_{0}=0.08 k^{-1}$ are shown in Fig. 4. The top and bottom rows are as in Fig. 3. The times shown in each panel are representative of the wave packet at early, pre-, and postoverturning times. From initialization through the majority of their evolution, the waves exhibit behavior qualitatively similar to the evolution at early times of nonhydrostatic waves. In particular, the induced mean flow differentially advects the waves near the center of the wave packet [Fig. 4(b)]. The associated phase changes observed in the nonhydrostatic case are less pronounced in the vertical cross section for the hydrostatic wave packet [Fig. 4(e)]. Isopycnal surfaces (represented by black curves) are found 

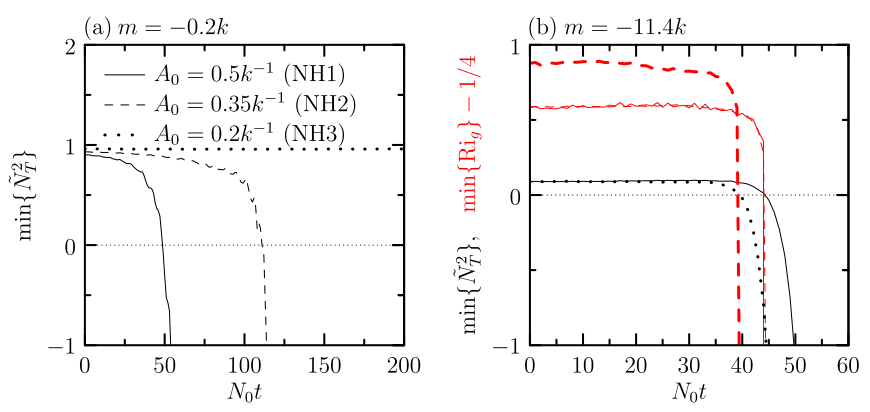

FIG. 5. Time series of $\min \left\{\tilde{N}_{T}^{2}\right\}$ (black curves), and $\min \left\{\mathrm{Ri}_{g}\right\}-1 / 4$ (red curves), in which $\tilde{N}_{T}^{2}$ and $\mathrm{Ri}_{g}$ are given respectively by Eqs. (28) and (19), for (a) strongly nonhydrostatic wave packets with $m=-0.2 k$ $\left(\Theta=11^{\circ}\right)$ with initial amplitudes $A_{0}=0.5 k^{-1}$ (NH1; solid curve), $A_{0}=0.35 k^{-1}$ (NH2; dashed curve), and $A_{0}=0.2 k^{-1}$ (NH3; heavy dotted curve), and (b) strongly hydrostatic wave packets with $m=-11.4 k(\Theta=$ $85^{\circ}$ ) and $A_{0}=0.08 k^{-1}$ with $f_{0}=0.01 N_{0}$ ( $\mathrm{H} 1$; solid curves) and $f_{0}=0.05 N_{0}$ ( $\mathrm{H}^{+}$; heavy dotted curves). The dashed red curve is a time series of $\min \left\{\mathrm{Ri}_{g}\right\}-1 / 4$ from $\mathrm{H} 1$ but using $\mathrm{Ri}_{g}=N_{T}^{2} /\left\|\left(\partial_{z} u, \partial_{z} v\right)\right\|^{2}$ as in Refs. [19,20].

to steepen sharply at the inflection point of the streamwise velocity where the vertical shear of the waves is largest. However, the streamwise-localized nature of the steepening is more characteristic of nonlinear steepening than shear instability. Unlike nonhydrostatic wave packets (e.g., NH1), the vertical displacement amplitude decreased in time due to linear dispersion. The waves overturned nonetheless at $t=45 / N_{0}$. The waves continued to evolve nonlinearly until $t=62 / N_{0}$, at which point the growth of sub-grid-scale convective instabilities was too small to be reliably resolved by our code.

For comparison, a simulation identical to $\mathrm{H} 1$ but with $f_{0}=0.05 N_{0}$ was performed $\left(\mathrm{H} 1^{+}\right)$. The initial Bretherton flow induced by this wave packet was shown in Fig. 1(a). The evolution of this wave packet was qualitatively similar to that of $\mathrm{H} 1$ from initialization through wave overturning. Despite the peak vertical displacement amplitude decreasing in time due to linear dispersion, the waves overturned at $t=40 / N_{0}$ (nearly one buoyancy period earlier than those in H1) due to the increased magnitude of the Bretherton flow with $f_{0}=0.05 N_{0}$ compared to that with $0.01 N_{0}$. Isopycnal surfaces (not shown) exhibited nonlinear steepening similar to that found with $\mathrm{H} 1$, suggesting shear instability likewise did not drive the waves with $f_{0}=0.05 N_{0}$ to overturning.

\section{Overturning of localized wave packets}

The overturning of wave packets is diagnosed by the criterion $N_{T}^{2}<0$. For convenience, we define the nondimensional squared total buoyancy frequency by

$$
\tilde{N}_{T}^{2} \equiv \frac{N_{T}^{2}}{N_{0}^{2}} .
$$

Time series of $\min \left\{\tilde{N}_{T}^{2}\right\}$ (taken over the entire spatial domain) are shown in Fig. 5(a) for simulations with $m=-0.2 k$ and initial amplitudes $A_{0}=0.5 k^{-1}$ (NH1; solid curve), $A_{0}=0.35 k^{-1}$ (NH2; dashed curve), and $A_{0}=0.2 k^{-1}$ (NH3; heavy dotted curve). The approach to overturning is diagnosed by decreasing values of $\min \left\{\tilde{N}_{T}^{2}\right\}$ in time. The decrease occurs more rapidly if the initial amplitude is larger but still well below the overturning amplitude predicted by linear theory.

Figure $5(\mathrm{~b})$ shows time series of $\min \left\{\tilde{N}_{T}^{2}\right\}$ (solid black curve) and $\min \left\{\mathrm{Ri}_{g}\right\}-1 / 4$ (solid and dashed red curves) for a strongly hydrostatic round wave packet (H1) with $m=-11.4 k$ and $A_{0}=0.08 k^{-1}$. The evolution of $\min \left\{\tilde{N}_{T}^{2}\right\}$ shows little change until $N_{0} t \approx 30$ when nonlinear effects rapidly drive the wave packet to overturning. In comparison, the evolution of $\min \left\{\mathrm{Ri}_{g}\right\}-1 / 4$ (solid red curve) shows little change until just before overturning. The dashed red curve, showing a time 


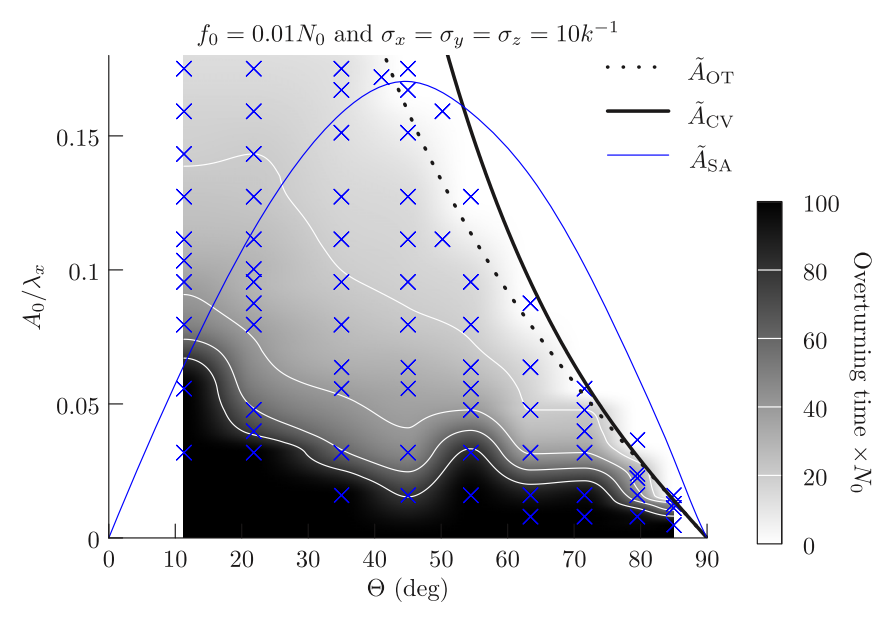

FIG. 6. Comparison of stability regimes with the results of simulations of round wave packets using initial vertical displacement amplitudes $A_{0} / \lambda_{x}$ and $\Theta=\tan ^{-1}(|m / k|)$ with rotation set by $f_{0}=0.01 N_{0}$. Each cross corresponds to the initial amplitude and wave number of a single simulation. Greyscale values represent overturning times, $t_{\mathrm{OT}}$, interpolated using cubic polynomials [28]. White curves are isolines of constant overturning time corresponding to $N_{0} t_{\mathrm{OT}}=20,40,60$, and 80 .

series of $\min \left\{\mathrm{Ri}_{g}\right\}-1 / 4$ using the definition of $\mathrm{Ri}_{g}$ in Refs. [19,20], evolves in a nearly identical manner. By either measure, the shear instability condition $\mathrm{Ri}_{g}<1 / 4$ was attained for the first time simultaneously as $\min \left\{\tilde{N}_{T}^{2}\right\}<0$. Likewise, these instability conditions were simultanously satisfied in time series of $\min \left\{\tilde{N}_{T}^{2}\right\}$ and $\min \left\{\mathrm{Ri}_{g}\right\}-1 / 4$ from $\mathrm{H} 1^{+}$(heavy dotted black and red curves, respectively). This suggests that shear instability alone cannot grow sufficiently quickly to overturn strongly hydrostatic waves, in agreement with previous predictions [23,24] and those in Sec. II B 2.

From a total of 79 simulations of round wave packets, the time to overturn was measured and compared with stability criteria as shown in Fig. 6. In all but the smallest amplitude cases, overturning occurred even though the initial wave amplitude was well below that predicted for overturning according to linear theory.

Waves with relative vertical wave number $m=-0.2 \mathrm{k}$ propagate at a shallow angle of $\Theta=11^{\circ}$ to the vertical. For this reason the waves spanning the range of small to moderately large initial amplitudes $\left(A_{0} / \lambda_{x}=0.096=1.48 \tilde{A}_{\mathrm{SA}}\right)$ were able to evolve without overturning until beyond $32 / N_{0}$. In simulations with larger $\Theta$, the maximum initial amplitude of waves that do not overturn until after $32 / N_{0}$ becomes smaller (relative to $\lambda_{x}$ ), but increases relative to $\tilde{A}_{\text {OT }}$. In particular, a hydrostatic wave packet $(\mathrm{H} 2)$ with $m=-5.4 k\left(\Theta \approx 80^{\circ}\right)$ and $A_{0} / \lambda_{x}=0.024=0.81 \tilde{A}_{\mathrm{OT}}$ overturned at $t=32 / N_{0}$, whereas one with $m=-11.4 k\left(\Theta=85^{\circ}\right)$ and $A_{0} / \lambda_{x}=0.013=0.91 \tilde{A}_{\mathrm{OT}}(\mathrm{H} 1)$ overturned at $t=45 / N_{0}$.

Simulations of long and wide wave packets required significantly larger computational resources and so only a limited series of simulations were performed with $m=-0.7 k$, initialized with $A_{0}=0.4 k^{-1}$ and $A_{0}=0.5 k^{-1}$, and with $m=-5.4 k$ and initial amplitude $A_{0}=0.15 k^{-1}$. These combinations were chosen as representative cases of nonhydrostatic waves in the transitional region between overturning before and after $32 / N_{0}$ (the cases with $m=-0.7 \mathrm{k}$ ), and of hydrostatic waves near the marginal stability value of $\tilde{A}_{\text {OT. }}$. The nonhydrostatic simulations of long wave packets (NH4-5), for which we set $\sigma_{x}=40 k^{-1}$ and $\sigma_{y}=10 k^{-1}\left(R_{y}=1 / 4\right)$, overturned within one $N_{0} t$ unit of the corresponding simulations of round wave packets. Wide wave packets in general are anticipated to interact with the Bretherton flow and induced long waves [9]. However, the wide wave packets reported on here were of such spanwise extent that induced long waves were predicted to be of negligible magnitude relative to the Bretherton flow. Simulations of wide wave packets, for which we set $\sigma_{x}=10 k^{-1}$ and $\sigma_{y}=40 k^{-1}\left(R_{y}=4\right)$, were generally less stable than corresponding round 
and long wave-packet simulations. For example, the case with $A_{0}=0.4 k^{-1}$ (NH6) overturned at $t=29 / N_{0}$, compared with the round (NH4) and long (NH7) wave packets, which both overturned at $t=32 / N_{0}$. This result contradicts the predicted increased stability of wide, compared to round, wave packets, shown in Fig. 2. The wave-packet aspect ratio appears to have had no effect on the results of the hydrostatic long and wide simulations. Wave packets initialized with $A_{0}=0.15 k^{-1}$ $\left(A_{0} / \lambda_{x}=0.024=0.81 \tilde{A}_{\mathrm{OT}}\right)$ were no more or less stable than their round counterparts. In particular, $\min \left\{\tilde{N}_{T}^{2}\right\}$ increased due to linear dispersion until $t \approx 13 / N_{0}$ (round and long wave packets; H2-3) and $t \approx 17 / N_{0}$ (wide wave packets; H4); before decreasing approximately monotonically until overturning at $t=32 / N_{0}$. These results, although spanning only a small region within the stability regimes shown in Fig. 2, are somewhat inconsistent with the prediction that long and wide wave packets are, respectively, less and more stable than their round counterparts. The open question regarding the stability and overturning of long and wide wave packets merits further study.

\section{DISCUSSION AND CONCLUSIONS}

Internal gravity waves are prone to instabilities resulting from overturning, convection whose onset is enhanced by self-acceleration. Strongly hydrostatic waves were found to not be prone to instability due to shear between adjacent, nearly horizontally aligned, velocity crests and troughs. To understand the instability processes that lead ultimately to wave breaking, we first derived an integral expression for the Bretherton flow induced by three-dimensional wave packets subject to Coriolis forces and performed fully nonlinear numerical simulations of localized wave packets with their Bretherton flow superimposed. We compared the initial amplitudes for which waves eventually overturned with the predictions of linear theory and characterized the results in terms of the instability mechanisms governing wave evolution.

We performed simulations of three-dimensional wave packets having wave numbers ranging from nonhydrostatic to near-inertial and amplitudes from small to overturning. Due to interactions with the mean flow induced by the waves, the waves were found to overturn even though their initial amplitude was well below the overturning amplitude, $\tilde{A}_{\mathrm{OT}}$, predicted by linear theory. Likewise, in all but the most strongly nonhydrostatic simulations, the self-acceleration condition overpredicted the amplitude for which wave packets eventually overturned. Strongly hydrostatic waves, propagating at angles $\Theta \gtrsim 80^{\circ}$, were found to overturn due to localized isopycnal steepening. Shear instability was not evident in time series of the minimum gradient Richardson number for strongly hydrostatic waves, in agreement with predictions.

Our results for round wave packets revealed that fully localized wave packets were unstable at lower amplitudes than their one- and two-dimensional analogs without rotation [14]. For example, a nonhydrostatic wave packet (NH8) with $m=-0.7 k$ and $A_{0}=0.6 k^{-1}$ overturned between $32 / N_{0}$ and $100 / N_{0}$ in the one-dimensional case, after $100 / N_{0}$ in the two-dimensional case, but at $t=21 / N_{0}$ in the three-dimensional case. The most strongly hydrostatic waves examined by Sutherland [14] $\left(\Theta=80^{\circ}\right)$ overturned after $100 / N_{0}$ even with initial amplitudes as large as $\tilde{A}_{\text {OT }}$ in both the one- and two-dimensional cases. Conversely, the equivalent three-dimensional wave packet with $A_{0}=0.15 k^{-1}\left(A_{0} / \lambda_{x}=0.024=0.81 \tilde{A}_{\text {OT }}\right)$ overturned at $t=32 / N_{0}$. We have shown that standard linear theory predictions for wave packet stability significantly over predict the amplitude at which overturning eventually occurs. This demonstrates that it is necessary to include the weakly nonlinear influence of the induced mean flow acting upon waves themselves. That said, at this time a theoretical prediction for the empirical results of fully nonlinear numerical simulations of fully three-dimensional wave packets remains elusive. Our results suggest the need for further theoretical and numerical investigations of wave-packet evolution.

As an application of our results, we contextualize our findings in terms of recent observations of internal gravity wave packets in the ocean. Therein, the internal wave frequency spectrum is dominated by strongly hydrostatic waves near the inertial frequency, $f_{0}$, forced by tidal flow over bathymetry and surface wind stress (e.g., Alford et al. [29]). However, turbulent breaking of nonhydrostatic waves has recently become of increasing interest for its potential role in larger 
scale oceanic processes, such as the global overturning circulation [30-33]. The Drake Passage has been identified as a source of lee waves, generated as the Antarctic Circumpolar Current flows over seamount chains. In particular, using two EM-APEX floats, Cusack et al. [30] measured a lee wave in the northernmost region of the Shackelton Fracture Zone (near $60^{\circ} \mathrm{S}$, with corresponding $\left.f_{0} \approx-1.26 \times 10^{-4} \mathrm{~s}^{-1}\right)$ with $A_{0}=100-140 \mathrm{~m}$ and $|m / k| \approx 0.6-2.5\left(\Theta \approx 32^{\circ}-68^{\circ}\right.$, hence $15.0 \gtrsim$ $\omega /\left|f_{0}\right| \gtrsim 6.5$ ). Assuming the wave to be monochromatic and evolving linearly, Cusack et al. [30] estimated $\lambda_{x}=4000 \mathrm{~m}$. No measurements were taken of the wave's spanwise structure. However, the strongest velocity perturbations were measured within $20 \mathrm{~km}$ of a prominent ridge feature of finite spanwise extent, suggesting the observed wave existed as a localized wave packet. On this basis, the observed wave corresponded to a wave packet with $A_{0} / \lambda_{x} \approx 0.10-0.14 \tilde{A}_{\mathrm{OT}}$ (for $|m / k| \approx$ 0.6 ) and $A_{0} / \lambda_{x} \approx 0.39-0.55 \tilde{A}_{\mathrm{OT}}$ (for $|m / k| \approx 2.5$ ) propagating in a fluid with $f_{0} / N_{0} \approx-0.06$ (using the reported local-mean buoyancy frequency, $N_{0} \approx 2.2 \times 10^{-3} \mathrm{rad} \mathrm{s}^{-1}$ ). For comparison, we consider a corresponding simulation (NH9-) with $|m / k|=2$ and $A_{0}=0.2 k^{-1}\left(\Theta \approx 63^{\circ}\right.$ and $A_{0} / \lambda_{x}=0.03=0.4 \tilde{A}_{\mathrm{OT}}$ ) and $f_{0} / N_{0}=-0.05$ (with corresponding $\omega /\left|f_{0}\right| \approx 9.0$ ). Setting $z_{0}=0$ and $t_{0}=0$ as the ridge top and time of observation, respectively, overturning was diagnosed at $(x, z)=(13.0,2.1) k^{-1}$ and $t=39 / N_{0} \approx 4.9 \mathrm{~h}$. Simulations with $f_{0} / N_{0}=0.01$ and 0.05 that were otherwise identical to $\mathrm{NH}^{-}{ }^{-}$resolved the same overturning time and location. The observed wave propagated against the Antarctic Circumpolar Current (with mean zonal velocity $33 \mathrm{~cm} \mathrm{~s}^{-1}$ ), hence the corresponding overturning site would be $x \approx 2.4 \mathrm{~km}$ upstream of and $z \approx 1.3 \mathrm{~km}$ above the ridge (or approximately $200 \mathrm{~m}$ below the ocean surface). Although the observed wave was not measured through its evolution, static and shear instability were proposed as playing a role in driving the wave eventually to overturning [30], despite remarking (as we have) that the initial amplitude was below both instability thresholds. Our results suggest the waves overturned due to nonlinear interactions with their induced mean flow. Time series of $\min \left\{\tilde{N}_{T}^{2}\right\}$ and $\min \left\{\operatorname{Ri}_{g}\right\}-1 / 4$ (not shown) indicate the simulated wave packet did not become shear unstable before overturning. This result was consistent across our simulated wave packets for all initial amplitudes and relative vertical wave numbers. Similarly, although beyond the scope of our numerical simulations, near-inertial wave packets with $\omega \gtrsim f_{0}$ induced by tidal flow over ridges [34] and by hurricanes [35] alike have been found to remain shear stable through their observed evolution, in agreement with predictions.

Although our simulations diagnosed overturning for a range of wave-packet parameters, the turbulent dynamics leading to wave breaking were not resolved, as this process evolves on small spatial and temporal scales that were prohibitively fine to be resolved by our numerical code. Furthermore, our results are highly idealized, having assumed an infinite, rotating but otherwise stationary medium under the Boussinesq approximation. Ongoing and future work is examining the evolution of fully localized moderately large amplitude wave packets in Boussinesq and anelastic fluids in which both background flow and buoyancy are nonuniform with height.

\section{ACKNOWLEDGMENTS}

The authors wish to thank WestGrid [36] and Compute Canada [37] for providing supercomputing resources, without which the fully nonlinear simulations would not have been possible. This research was supported by the Natural Science and Engineering Research Council (NSERC) of Canada. T.S.vdB. acknowledges a Royal Academy of Engineering Research Fellowship. Figures 1, 3 , and 4 used the cmocean color map [38].

[1] O. Bühler, Waves and Mean Flows (Cambridge University Press, Cambridge, UK, 2009), p. 341.

[2] B. R. Sutherland, Internal Gravity Waves (Cambridge University Press, Cambridge, UK, 2010), p. 378.

[3] T. G. Shepherd, Symmetries, conservation laws, and Hamiltonian structure in geophysical fluid dynamics, Adv. Geophys. 32, 287 (1990). 
[4] J. F. Scinocca and T. G. Shepherd, Nonlinear wave-activity conservation laws and Hamiltonian structure for the two-dimensional anelastic equations. J. Atmos. Sci. 49, 5 (1992).

[5] F. P. Bretherton, On the mean motion induced by gravity waves, J. Fluid Mech. 36, 785 (1969).

[6] A. Tabaei and T. R. Akylas, Resonant long-short wave interactions in an unbounded rotating stratified fluid, Stud. Appl. Maths 119, 271 (2007).

[7] T. S. van den Bremer and B. R. Sutherland, The mean flow and long waves induced by two-dimensional internal gravity wavepackets, Phys. Fluids 26, 106601 (2014).

[8] B. R. Sutherland, W. Reeves, and T. S. van den Bremer, Flows induced by Coriolis-influenced vertically propagating two-dimensional internal gravity wave packets, Phys. Rev. Fluids 5, 064805 (2020).

[9] T. S. van den Bremer and B. R. Sutherland, The wave-induced flow of wide three-dimensional internal gravity wavepackets, J. Fluid Mech. 834, 385 (2018).

[10] T. R. Akylas and A. Tabaei, Resonant self-acceleration and instability of nonlinear internal gravity wavetrains, in Frontiers of Nonlinear Physics, edited by A. Litvak (Institute of Applied Physics, Nizhny Novgorod, Russia, 2005), pp. 129.

[11] B. R. Sutherland, Weakly nonlinear internal gravity wavepackets, J. Fluid Mech. 569, 249 (2006).

[12] G. B. Whitham, Linear and Nonlinear Waves (John Wiley and Sons, Inc., New York, 1974), p. 636.

[13] A. D. Gervais, G. E. Swaters, T. S. van den Bremer, and B. R. Sutherland, Evolution and stability of two-dimensional anelastic internal gravity wave packets, J. Atmos. Sci. 75, 3703 (2018).

[14] B. R. Sutherland, Finite-amplitude internal wavepacket dispersion and breaking, J. Fluid Mech. 429, 343 (2001).

[15] O. Bühler and M. E. McIntyre, On non-dissipative wave-mean interactions in the atmosphere or oceans, J. Fluid Mech. 354, 301 (1998).

[16] O. Bühler and M. E. McIntyre, Remote recoil: A new wave-mean interaction effect, J. Fluid Mech. 492, 207 (2003).

[17] G. L. Wagner and W. R. Young, Available potential vorticity and wave-averaged quasi-geostrophic flow, J. Fluid Mech. 785, 401 (2015).

[18] J.-H. Xie and J. Vanneste, A generalised-Lagrangian-mean model of the interactions between near-inertial waves and mean flow, J. Fluid Mech. 774, 143 (2015).

[19] D. C. Fritts and P. K. Rastogi, Convective and dynamical instabilities due to gravity wave motions in the lower and middle atmosphere: Theory and observations, Radio Sci. 20, 1247 (1985).

[20] U. Achatz, Gravity-wave breaking: Linear and primary nonlinear dynamics, Adv. Space. Res. 40, 719 (2007).

[21] J. W. Miles, On the stability of heterogeneous shear flows, J. Fluid Mech. 10, 496 (1961).

[22] L. N. Howard, Note on a paper by John W. Miles, J. Fluid Mech. 10, 509 (1961).

[23] E. E. Gossard, D. R. Jensen, and J. H. Richter, An analytical study of tropospheric structure as seen by high-resolution radar, J. Atmos. Sci. 28, 794 (1971).

[24] J. Klostermeyer, Two-dimensional and three-dimensional parametric instabilities in finite amplitude internal gravity waves, Geophys. Astrophys. Fluid Dyn. 61, 1 (1991).

[25] H. V. Dosser and B. R. Sutherland, Anelastic internal wavepacket evolution and stability, J. Atmos. Sci. 68, 2844 (2011).

[26] J. H. Williamson, Low-storage Runge-Kutta schemes, J. Comp. Phys. 35, 48 (1980).

[27] D. R. Durran, Numerical Methods for Fluid Dynamics, 2nd ed. (Springer-Verlag, New York, 2010), p. 516.

[28] M. Steffen, A simple method for monotonic interpolation in one dimension, Astron. Astrophys. 239, 443 (1990).

[29] M. H. Alford, J. A. MacKinnon, H. L. Simmons, and J. D. Nash, Near-inertial internal gravity waves in the ocean, Annu. Rev. Mar. Sci. 8, 95 (2016).

[30] J. M. Cusack, A. C. Naveira Garabato, D. A. Smeed, and J. B. Girton, Observation of a large lee wave in the Drake Passage, J. Phys. Oceanogr. 47, 793 (2017).

[31] L. D. Talley, Closure of the global overturning circulation through the Indian, Pacific, and Southern Oceans: Schematics and transports, Oceanography 26, 80 (2013). 
[32] A. Melet, R. Hallberg, S. Legg, and M. Nikurashin, Sensitivity of the ocean state to lee wave-driven mixing, J. Phys. Oceanogr. 44, 900 (2014).

[33] A. F. Waterhouse, J. A. MacKinnon, J. D. Nash, M. H. Alford, E. Kunze, H. L. Simmons, K. L. Polzin, L. C. St. Laurent, O. M. Sun, R. Pinkel, L. D. Talley, C. B. Whalen, T. N. Huussen, G. S. Carter, I. Fer, S. Waterman, A. C. Naveira Garabato, T. B. Sanford, and C. M. Lee, Global patterns of diapycnal mixing from measurements of the turbulent dissipation rate, J. Phys. Oceanogr. 44, 1854 (2014).

[34] M. H. Alford, Sustained, full-water-column observations of internal waves and mixing near Mendocino Escarpment, J. Phys. Oceanogr. 40, 2643 (2010).

[35] T. B. Sanford, J. F. Price, and J. B. Girton, Upper-ocean response to Hurricane Frances (2004) observed by profiling EM-APEX floats, J. Phys. Oceanogr. 41, 1041 (2011).

[36] www.westgrid.ca.

[37] www.computecanada.ca.

[38] K. M. Thyng, C. A. Greene, R. D. Hetland, H. M. Zimmerle, and S. F. DiMarco, True colors of oceanography: Guidelines for effective and accurate colormap selection, Oceanography 29, 9 (2016). 